TRANSACTIONS OF THE

AMERICAN MATHEMATICAL SOCIETY

Volume 351, Number 6, Pages 2213-2232

S 0002-9947(99)02143-1

Article electronically published on February 23, 1999

\title{
THE DIAGONAL SUBRING AND THE COHEN-MACAULAY PROPERTY OF A MULTIGRADED RING
}

\author{
EERO HYRY
}

\begin{abstract}
Let $T$ be a multigraded ring defined over a local ring $(A, \mathfrak{m})$. This paper deals with the question how the Cohen-Macaulay property of $T$ is related to that of its diagonal subring $T^{\Delta}$. In the bigraded case we are able to give necessary and sufficient conditions for the Cohen-Macaulayness of $T$. If $I_{1}, \ldots, I_{r} \subset A$ are ideals of positive height, we can then compare the CohenMacaulay property of the multi-Rees algebra $R_{A}\left(I_{1}, \ldots, I_{r}\right)$ with the CohenMacaulay property of the usual Rees algebra $R_{A}\left(I_{1} \cdots I_{r}\right)$. We also obtain a bound for the joint reduction numbers of two m-primary ideals in the case the corresponding multi-Rees algebra is Cohen-Macaulay.
\end{abstract}

\section{INTRODUCTION}

Let $(A, \mathfrak{m})$ be a local ring. Let $T=\bigoplus_{n \in \mathbb{N}^{r}} T_{n}$ be a multigraded ring finitely generated over $A$ by elements in degrees $(1,0, \ldots, 0), \ldots,(0, \ldots, 0,1)$. In this paper we are interested in studying the relationship between the Cohen-Macaulay property of $T$ and that of its diagonal subring $T^{\Delta}$ which is the graded $\operatorname{ring} T^{\Delta}=\bigoplus_{n \in \mathbb{N}} T_{n, \ldots, n}$. The geometric object associated to a multigraded ring $T$ is the corresponding multiprojective scheme Proj $T$ constructed by means of multihomogeneous localizations. It is easy to see that $\operatorname{Proj} T$ is isomorphic to the usual projective scheme $\operatorname{Proj} T^{\Delta}$. From this point of view it is natural to expect that the homological properties of $T$ and $T^{\Delta}$ are closely related. Classically $T$ is the multihomogeneous coordinate ring of a multiprojective variety $V$ defined over a field $k$ and contained in some multiprojective space $\mathbb{P}_{k}^{n_{1}} \times \cdots \times \mathbb{P}_{k}^{n_{r}}$. The ring $T^{\Delta}$ is then the homogeneous coordinate ring of the image of $V$ in the Segre imbedding $\mathbb{P}_{k}^{n_{1}} \times \cdots \times \mathbb{P}_{k}^{n_{r}} \rightarrow \mathbb{P}_{k}^{N}$ where $N=\left(n_{1}+1\right) \cdots\left(n_{r}+1\right)-1$.

A class of multigraded rings of special interest to us is that of multi-Rees algebras. Let $I_{1}, \ldots, I_{r} \subset A$ be ideals of positive height. The multi-Rees algebra $R_{A}\left(I_{1}, \ldots, I_{r}\right)=A\left[I_{1} t_{1}, \ldots, I_{r} t_{r}\right]$ where $t_{1}, \ldots, t_{r}$ are indeterminates. Geometrically multi-Rees algebras arise by successive blowing up: $Z_{j}=\operatorname{Proj} R_{A}\left(I_{1}, \ldots, I_{j}\right)$ is for all $j=1, \ldots, r$ the blow-up of $Z_{j-1}=\operatorname{Proj} R_{A}\left(I_{1}, \ldots, I_{j-1}\right)$ along the closed subscheme determined by the sheaf of ideals $I_{j} \mathcal{O}_{Z_{j-1}}$. The diagonal subring is now the usual Rees algebra $R_{A}\left(I_{1} \cdots I_{r}\right)$ of the product $I_{1} \cdots I_{r}$. We shall show in Corollary 2.10 of this paper that if $R_{A}\left(I_{1}, \ldots, I_{r}\right)$ is Cohen-Macaulay, then so is also $R_{A}\left(I_{1} \cdots I_{r}\right)$. This is a consequence of our main Theorem 2.5 , where we give nec-

Received by the editors June 1, 1996

1991 Mathematics Subject Classification. Primary 13A30; Secondary 14B15, 14M05.

(C)1999 American Mathematical Society 
essary and sufficient conditions for the Cohen-Macaulayness of a general bigraded ring $T$ with negative $a$-invariants $a^{1}(T)$ and $a^{2}(T)$. In the case $T=R_{A}(I, J)$ where $I, J \subset A$ are ideals of positive height, these conditions mean that the CohenMacaulay property of $T$ can be characterized in terms of the local cohomologies of the ideals $I^{p} R_{A}(I J), J^{p} R_{A}(I J) \subset R_{A}(I J)(p \in \mathbb{N})$. We show by example that the converse of Corollary 2.10 does not hold. In Theorem 2.13 we consider conditions under which the Cohen-Macaulayness of $R_{A}(I J)$ implies that of $R_{A}\left(I^{k}, J^{l}\right)$ for $k, l \gg 0$. Our results generalize the results of [6] and [7], where the properties of multi-Rees algebras were studied under the assumption that the ideals $I_{1}, \ldots, I_{r}$ are all powers of the same ideal.

As a classical application of Theorem 2.5 we consider a $\operatorname{ring} T=S / I$ where $S=A\left[X_{1}, \ldots, X_{r}, Y_{1}, \ldots, Y_{s}\right]$ is a polynomial ring defined over a local CohenMacaulay ring $A$ and $I \subset S$ is a bihomogeneous ideal. Suppose that $I$ can be generated by forms of bidegree $(d, e)$ with $d, e>0$ and that ht $I<\min (r / d, s / e)$. It turns out in Theorem 2.14 that if $T$ is Cohen-Macaulay, then also the diagonal $\operatorname{ring} T^{\Delta}$ is Cohen-Macaulay. This problem has recently been investigated by Trung, Simis and Valla in the case $A=k$ is a field and $T$ is a Rees algebra of a homogeneous ideal in $k\left[X_{1}, \ldots, X_{r}\right]$ (cf. [19]).

Recall that if $I \subset A$ is an ideal, an ideal $J \subset I$ is said to be a reduction of $I$ if $I^{n+1}=J I^{n}$ for $n \gg 0$. The reduction number $r_{J}(I)$ with respect to $J$ is the least integer $n$ satisfying this condition. Reductions were first defined in [15] and have proved important in finding out conditions which make a usual Rees algebra CohenMacaulay. The concept of a reduction of an ideal can be generalized to the case of several ideals. Rees introduced in [17] the notions of complete and joint reductions. More general definitions have later been given in [11]. Let $I, J \subset A$ be ideals. A set $K=\left\{a_{1}, \ldots, a_{\lambda}, b_{1}, \ldots, b_{\lambda}\right\}$ where $a_{1}, \ldots, a_{\lambda} \in I$ and $b_{1}, \ldots, b_{\lambda} \in J$, is termed a complete reduction of $I$ and $J$ if $(I J)^{n+1}=\left(a_{1} b_{1}, \ldots, a_{\lambda} b_{\lambda}\right) I^{n} J^{n}$ for $n \gg 0$. If the residue field of $A$ is infinite, there exists complete reductions with $\lambda=l(I J)$ where $l(I J)$ denotes the analytic spread of $I J([16])$. Let $\mu+\nu=\lambda$. Consider a partition of the set $\{1, \ldots, \lambda\}$ into two disjoint subsets $\left\{i_{1}, \ldots, i_{\mu}\right\}$ and $\left\{j_{1}, \ldots, j_{\nu}\right\}$. The subset $\left\{a_{i_{1}}, \ldots, a_{i_{\mu}}, b_{j_{1}}, \ldots, b_{j_{\nu}}\right\} \subset K$ then forms a joint reduction of $I$ and $J$ of type $(\mu, \nu)$ meaning that $(I J)^{n+1}=\left(a_{i_{1}}, \ldots, a_{i_{\mu}}\right) I^{n} J^{n+1}+\left(b_{j_{1}}, \ldots, b_{j_{\nu}}\right) I^{n+1} J^{n}$ for $n \gg 0$. We call the smallest integer $n$ satisfying this condition for all partitions $\left\{i_{1}, \ldots, i_{\mu}\right\} \cup\left\{j_{1}, \ldots, j_{\nu}\right\}$ of $\{1, \ldots, \lambda\}$ the joint reduction number of $I$ and $J$ of type $(\mu, \nu)$ with respect to $K$ and denote it by $r_{K}^{\mu, \nu}(I, J)$. Suppose now that the ideals $I$ and $J$ are $\mathfrak{m}$-primary and that $\lambda=\operatorname{dim} A$. We can prove in Corollary 3.4 that if $R_{A}(I, J)$ is Cohen-Macaulay, then $r_{K}^{\mu, \nu}(I, J) \leq \max (\mu, \nu)-1$ for all $\mu, \nu \geq 1$ with $\mu+\nu=\operatorname{dim} A$. This generalizes the well-known result saying that if $I \subset A$ is an $\mathfrak{m}$-primary ideal such that $R_{A}(I)$ is Cohen-Macaulay, then $r_{J}(I) \leq \operatorname{dim} A-1$ for every $\operatorname{dim} A$-generated reduction $J \subset I$ (cf. [21, Theorem 4.2]).

In the two-dimensional case Verma investigated in [25] the Cohen-Macaulay property of multi-Rees algebras of $\mathfrak{m}$-primary ideals under the assumption that the joint reduction numbers of the ideals are zero. Later this situation was considered also in [8]. Corollary 3.4 now implies that the sufficient condition given in [8] is also necessary: If $A$ is a local Cohen-Macaulay ring of dimension two and $I, J \subset A$ are $\mathfrak{m}$-primary ideals, then $R_{A}(I, J)$ is Cohen-Macaulay if and only if the usual Rees algebras $R_{A}(I), R_{A}(J)$ are Cohen-Macaulay and the mixed multiplicity $e_{1}(I, J)=l(A / I J)-l(A / I)-l(A / J)($ cf. Corollary 3.5). 


\section{Preliminaries}

We begin by recalling some basic facts about multigraded rings and modules. For more details we refer to [3] (cf. also [1] and [9]). Let $T=\bigoplus_{n \in \mathbb{Z}^{r}} T_{\mathbf{n}}$ be an $r$-graded ring defined over a ring $A=T_{0}$. We always assume that all rings are Noetherian. The ring $T$ has certain subrings important to us. The diagonal subring of $T$ is the graded ring $T^{\Delta}=\bigoplus_{n \in \mathbb{Z}} T_{n, \ldots, n}$. For any $j \in\{1, \ldots, r\}$, there is an $r-1$-graded subring $T_{\ldots, 0, \ldots}{ }^{(j)}=\bigoplus_{n_{j}=0} T_{\mathbf{n}}$. If $M$ is an $r$-graded $T$-module, we then have a graded $T^{\Delta}$-module $M^{\Delta}=\bigoplus_{n \in \mathbb{Z}} M_{n, \ldots, n}$ and $r$ - 1 -graded $T \ldots, 0, \ldots$-modules $M_{\ldots, k, \ldots}=\bigoplus_{n_{j}=k} M_{\mathbf{n}}(k \in \mathbb{Z})$. When $T$ is $\mathbb{N}^{r}$-graded and $A$ is local with the maximal ideal $\mathfrak{m}, T$ has a unique homogeneous maximal ideal $\mathfrak{M}=\mathfrak{m} \oplus \bigoplus_{\mathbf{n} \neq \mathbf{0}} T_{\mathbf{n}}$. The homogeneous maximal ideals of $T^{\Delta}$ and $T_{\ldots, 0, \ldots}$ are then $\mathfrak{M}^{\Delta}$ and $\mathfrak{M}_{\ldots, 0, \ldots}$ respectively.

If $\mathfrak{A} \subset T$ is a homogeneous ideal, the local cohomology functors $\underline{H}_{\mathfrak{A}}^{i}(\cdot)$ are defined in the category of $r$-graded $T$-modules as usual. As $T$-modules $r$-graded local cohomology modules of course coincide with the usual ones. In many occasions it is useful to consider the ring $T$ endowed with a different grading. Local cohomology modules behave well under a change of grading. To put this more precisely, given a homomorphism $\varphi: \mathbb{Z}^{r} \rightarrow \mathbb{Z}^{q}$, set

$$
M^{\varphi}=\bigoplus_{\mathbf{m} \in \mathbb{Z}^{q}}\left(\bigoplus_{\varphi(\mathbf{n})=\mathbf{m}} M_{\mathbf{n}}\right)
$$

for any $r$-graded $T$-module $M$. Then $T^{\varphi}$ is a $q$-graded ring and $M^{\varphi}$ a $q$-graded $T^{\varphi}$-module. We now have the result that $\left(\underline{H}_{\mathfrak{A}}^{i}(M)\right)^{\varphi}=\underline{H}_{\mathfrak{Q} \varphi}^{i}\left(M^{\varphi}\right)$. To see this, it is enough to note that $\left(\underline{H}_{\mathfrak{A}}^{i}(\cdot)\right)^{\varphi}$ and $\underline{H}_{\mathfrak{A} \varphi}^{i}(\cdot \varphi)$ are both universal $\delta$-functors, which coincide if $i=0$. We are often interested in vanishing of local cohomology. It is then useful to observe the following simple fact:

1.1. Lemma. Let $T$ be an r-graded ring defined over a local ring $(A, \mathfrak{m})$. Set $S=T_{\ldots, 0, \ldots}$ and $\mathfrak{N}=\mathfrak{M}_{\ldots, 0, \ldots}$. Consider $T_{\mathfrak{N}}$ as a graded ring defined over the local ring $S_{\mathfrak{N}}$. Then $\left[\underline{H}_{\mathfrak{A}_{\mathfrak{N}}}^{i}\left(T_{\mathfrak{N}}\right)\right]_{k}=0$ if and only if $\left[\underline{H}_{\mathfrak{A}}^{i}(T)\right]_{\mathbf{n}}=0$ for all $\mathbf{n} \in \mathbb{Z}^{r}$ with $n_{j}=k$.

Proof. The above mentioned result implies that if $T$ is considered as a graded $S$ algebra, then $\left[\underline{H}_{\mathfrak{A}}^{i}(T)\right]_{k}=\bigoplus_{n_{j}=k}\left[\underline{H}_{\mathfrak{A}}^{i}(T)\right]_{\mathbf{n}}$ is a graded $S$-module for every $k \in \mathbb{Z}$. This implies the claim, since $\left[\underline{H}_{\mathfrak{A}_{\mathfrak{N}}}^{i}\left(T_{\mathfrak{N}}\right)\right]_{k}=\left(\left[\underline{H}_{\mathfrak{A}}^{i}(T)\right]_{k}\right)_{\mathfrak{N}}$.

If $T$ is $\mathbb{N}^{r}$-graded and can be finitely generated over $A$ by elements in degrees $(1,0, \ldots, 0), \ldots,(0, \ldots, 0,1)$, we call $T$ standard. Suppose from now on that $T$ is standard. The irrelevant ideal of $T$ is $T^{+}=\bigoplus_{n_{1}, \ldots, n_{r}>0} T_{\mathbf{n}}$. Also set $T_{j}^{+}=$ $\bigoplus_{n_{j}>0} T_{\mathbf{n}}$ for all $j=1, \ldots, r$. Let Proj $T$ denote the set of all homogeneous prime ideals $P \subset T$ which do not contain $T^{+}$. Consider $\operatorname{Proj} T$ as a topological space with closed subsets $V_{+}(I)=\{P \in \operatorname{Proj} T \mid P \supset I\}$ where $I \subset T$ is a homogeneous ideal. We give Proj $T$ a scheme structure analogously to the usual graded case by using homogeneous localizations

$$
T_{(P)}=\left\{\frac{a}{f} \mid a, f \in T_{\mathbf{n}}, f \notin P, \mathbf{n} \in \mathbb{N}^{r}\right\} \quad(P \in \operatorname{Proj} T) .
$$

We call $T$ the $r$-projective scheme corresponding to $T$. The theory of multiprojective schemes is similar to that of projective schemes, which can be found in [4]. We 
therefore mainly list here some most important facts. Set $Z=\operatorname{Proj} T$. There is a canonical projection $Z \rightarrow \operatorname{Spec} A$, which is a proper morphism. If $M$ is an $r$ graded $T$-module, we have the associated quasi-coherent sheaf $\mathcal{M}$ of $\mathcal{O}_{Z}$-modules. The quasi-coherent sheaf corresponding to $T(\mathbf{n})$ is invertible for every $\mathbf{n} \in \mathbb{Z}^{r}$. We denote it by $\mathcal{O}_{Z}(\mathbf{n})$. Moreover, we have $\mathcal{O}_{Z}(\mathbf{m}+\mathbf{n})=\mathcal{O}_{Z}(\mathbf{m}) \otimes \mathcal{O}_{Z}(\mathbf{n})$ for all $\mathbf{m}, \mathbf{n} \in \mathbb{Z}^{r}$. Multiprojective schemes are projective: if $Z^{\Delta}=\operatorname{Proj} T^{\Delta}$, the inclusion $T^{\Delta} \rightarrow T$ induces an isomorphism $f: Z \rightarrow Z^{\Delta}$ such that $f^{*}\left(\mathcal{O}_{Z^{\Delta}}(n)\right)=$ $\mathcal{O}_{Z}(n, \ldots, n)$ for all $n \in \mathbb{Z}$. In the next lemma we consider the dimension of $Z$.

\subsection{Lemma.}

1) We have $\operatorname{dim} Z=\sup \{\operatorname{dim} T / P \mid P \in Z \cap \operatorname{Min} T\}-r$. It follows, in particular, that if $\operatorname{dim} T_{\ldots, 0, \ldots}{ }^{(j)}<\operatorname{dim} T$ for all $j=1, \ldots, r$, then $\operatorname{dim} Z=\operatorname{dim} T-r$.

2) If $T$ is a catenary domain and $A$ is local, then $\operatorname{dim} \mathcal{O}_{Z, P}=\operatorname{dim} Z$ for all closed points $P \in Z$.

Proof. Let $P \in Z$ be a closed point. The projection $Z \rightarrow \operatorname{Spec} A$ being proper, $P_{\mathbf{0}}=P \cap A$ is a closed point of $\operatorname{Spec} A$. Then $(T / P)_{\mathbf{0}}$ is a field. Moreover, we have $\operatorname{dim} \operatorname{Proj} T / P=0$. This implies that $\operatorname{dim} T / P=r$ : it is easy to check that $0 \subset S_{1}^{+} \subset S_{1}^{+}+S_{2}^{+} \subset \cdots \subset S_{1}^{+}+\cdots+S_{r}^{+}$is a maximal chain of homogeneous prime ideals of $S=T / P$. Any maximal chain of homogeneous prime ideals of $T$ starting from a minimal prime $P_{0} \in Z$ is then of type $P_{0} \subset \cdots \subset P_{n} \subset \cdots \subset P_{n+r}$ where $P_{n}$ is a closed point of $Z$. As $\operatorname{dim} Z=\sup \{$ ht $P \mid P \in Z\}$, the formula $\operatorname{dim} Z=\sup \{\operatorname{dim} T / P \mid P \in Z \cap \operatorname{Min} T\}-r$ follows. Since $T_{1}^{+} \cdots T_{r}^{+} \subset T^{+}$, the condition $\operatorname{dim} T / T_{j}^{+}<\operatorname{dim} T(j=1, \ldots, r)$ means that $Z$ contains all minimal primes $P$ of $T$ with $\operatorname{dim} T / P=\operatorname{dim} T$. Then $\operatorname{dim} Z=\operatorname{dim} T-r$ proving 1$)$. In the case of 2) we always have above $P_{0}=0$ and $P_{n+r}=\mathfrak{M}$. Since $T$ is catenary, this implies for any closed point $P \in Z$ that $\operatorname{dim} \mathcal{O}_{Z, P}=\operatorname{ht} P=\operatorname{dim} T-r=\operatorname{dim} Z$.

We can look at the scheme $Z$ also from another point of view. Take $j \in\{1, \ldots, r\}$ and let $Y$ denote the $r-1$-projective scheme $\operatorname{Proj} T_{\ldots, 0, \ldots}{ }^{(j)}$. For every $k \in \mathbb{N}$, let $\mathcal{T}_{k}$ be the quasi-coherent $\mathcal{O}_{Y}$-module corresponding to the $r-1$-graded $T_{\ldots, 0, \ldots}$-module $T_{\ldots, k, \ldots}$. Then $\mathcal{T}=\bigoplus_{k \geq 0} \mathcal{T}_{k}$ is a quasi-coherent graded $\mathcal{O}_{Y}$-algebra so that we have an associated projective scheme $\operatorname{Proj} \mathcal{T}$. Recall that $\operatorname{Proj} \mathcal{T}$ can be constructed by glueing together the schemes Proj $\bigoplus_{k \geq 0}\left(T_{\ldots, k, \ldots}\right)_{(b)}$ where $b \in T_{1, \ldots, 1,0,1, \ldots, 1}$. It is now not difficult to see that it is possible to identify $\operatorname{Proj} \mathcal{T}$ with $Z$. The corresponding canonical invertible sheaf on $Z$ is $\mathcal{O}_{Z}(0, \ldots, 0,1,0, \ldots, 0)$. Moreover, if $g: Z \rightarrow Y$ is the canonical projection, we have

$$
\mathcal{O}_{Z}\left(n_{1}, \ldots, n_{j-1}, 0, n_{j+1}, \ldots, n_{r}\right)=g^{*}\left(\mathcal{O}_{Y}\left(n_{1}, \ldots, n_{j-1}, n_{j+1}, \ldots, n_{r}\right)\right)
$$

for $n_{1}, \ldots, n_{j-1}, n_{j+1}, \ldots, n_{r} \in \mathbb{Z}$. If $M$ is an $r$-graded $T$-module and $\mathcal{M}_{k}$ is the quasi-coherent $\mathcal{O}_{Y}$-module corresponding to the $r-1$-graded $T_{\ldots, 0, \ldots}$-module $M_{\ldots, k, \ldots}(k \in \mathbb{Z})$, then $\mathcal{M}$ is the quasi-coherent $\mathcal{O}_{Z}$-module corresponding to the graded $\mathcal{O}_{Y}$-module $\bigoplus_{k \in \mathbb{Z}} \mathcal{M}_{k}$. There is for all $\mathbf{n} \in \mathbb{Z}^{r}$ a canonical homomorphism

$$
\mathcal{M}_{n_{j}}\left(n_{1}, \ldots, n_{j-1}, n_{j+1}, \ldots, n_{r}\right) \rightarrow g_{*}(\mathcal{M}(\mathbf{n})) \text {. }
$$

Let $\mathfrak{a} \subset A$ be an ideal. We want to compare the multigraded local cohomology with respect to the ideal $\left(\mathfrak{a}, T^{+}\right) \subset T$ with the sheaf cohomology with supports in $E=V_{+}(\mathfrak{a} T) \subset Z$. To this purpose we need a multigraded variant of the so called Sancho de Salas sequence ([14]). First we prove the following lemma: 
1.3. Lemma. Let I be an injective $r$-graded $T$-module and let $\mathcal{I}$ be the corresponding quasi-coherent sheaf of $\mathcal{O}_{Z}$-modules. Then $\mathcal{I}$ is an injective $\mathcal{O}_{Z}$-module.

Proof. It is enough to show that $I_{(P)}$ is an injective $T_{(P)}$-module for all $P \in Z$ (cf. [5, Chapter II, Proposition 7.17]). For any $T$-module $N$, set

$$
N_{\langle P\rangle}=\left\{\frac{x}{f} \mid x \in N, f \in T \backslash P \text { is homogeneous }\right\} .
$$

It is easy to check that $I_{\langle P\rangle}$ is an injective $r$-graded $T_{\langle P\rangle}$-module. The claim now follows from the fact that the category of $r$-graded $T_{\langle P\rangle}$-modules is equivalent to the category of $T_{(P)}$-modules. The equivalence is given by $M \mapsto M_{\mathbf{0}}$. Indeed, according to the $r$-graded version of $[9$, Proposition $(12.17)] T_{\langle P\rangle} \cong T_{(P)}\left[t_{1}, \ldots, t_{r}, t_{1}^{-1}, \ldots, t_{r}^{-1}\right]$ where $t_{1}, \ldots, t_{r}$ are indeterminates. Then, for any $r$-graded $T_{\langle P\rangle}$-module $M$, we have $M \cong M_{0}\left[t_{1}, \ldots, t_{r}, t_{1}^{-1}, \ldots, t_{r}^{-1}\right]$.

1.4. Theorem. Let $T$ be a standard $r$-graded ring defined over a ring $A$ and let $\mathfrak{a} \subset A$ be an ideal. Let $M$ be an r-graded T-module. With the preceding notation, there exists an exact sequence

$$
\cdots \rightarrow \underline{H}_{\left(\mathfrak{a}, T^{+}\right)}^{i}(M) \rightarrow \bigoplus_{\mathbf{n} \in \mathbb{Z}^{r}} H_{\mathfrak{a}}^{i}\left(M_{\mathbf{n}}\right) \rightarrow \bigoplus_{\mathbf{n} \in \mathbb{Z}^{r}} H_{E}^{i}(Z, \mathcal{M}(\mathbf{n})) \rightarrow \cdots
$$

of $r$-graded T-modules. Moreover, if $j \in\{1, \ldots, r\}$ and $S=T_{\ldots, 0, \ldots}^{(j)}$, we have a commutative diagram of r-graded $T$-modules



where $F=V_{+}(\mathfrak{a} S), \hat{\mathbf{n}}=\left(n_{1}, \ldots, n_{j-1}, n_{j+1}, \ldots, n_{r}\right)$ and the homomorphisms

$$
H_{F}^{i}\left(Y, \mathcal{M}_{n_{j}}(\hat{\mathbf{n}})\right) \rightarrow H_{E}^{i}(Z, \mathcal{M}(\mathbf{n}))
$$

are composites

$$
H_{F}^{i}\left(Y, \mathcal{M}_{n_{j}}(\hat{\mathbf{n}})\right) \rightarrow H_{F}^{i}\left(Y, g_{*}(\mathcal{M}(\mathbf{n}))\right) \rightarrow H_{E}^{i}(Z, \mathcal{M}(\mathbf{n}))
$$

of canonical homomorphisms.

Proof. The construction of the multigraded Sancho de Salas sequence is analogous to that of the usual Sancho de Salas sequence. We recall this construction following an idea sketched in [14, p. 2]. Set $C=V\left(T^{+}\right) \subset \operatorname{Spec} T$ and $U=\operatorname{Spec} T \backslash C$. Let $0 \rightarrow M \rightarrow I^{\bullet}$ be an $r$-graded injective resolution of $M$. As $H_{C}^{1}\left(\operatorname{Spec} T, \widetilde{I}^{p}\right)=$ $\underline{H}_{T^{+}}^{1}\left(I^{p}\right)=0$ for all $p \in \mathbb{N}$ we obtain an exact sequence

$$
0 \rightarrow \Gamma_{C}\left(\operatorname{Spec} T, \widetilde{I}^{\bullet}\right) \rightarrow I^{\bullet} \rightarrow \Gamma\left(U, \widetilde{I}^{\bullet}\right) \rightarrow 0
$$

where $\Gamma_{C}\left(\operatorname{Spec} T, \widetilde{I}^{\bullet}\right)=\underline{H}_{T^{+}}^{0}\left(I^{\bullet}\right)$. There is an affine morphism $h: U \rightarrow Z$ taking a prime ideal $P \in U$ to its homogenization $P^{*} \in Z$. If $N$ is an $r$-graded $T$-module and $\mathcal{N}$ the corresponding quasi-coherent $\mathcal{O}_{Z}$-module, we clearly have $h_{*}\left(\widetilde{N}_{\mid U}\right)=$ $\bigoplus_{\mathbf{n} \in \mathbb{Z}^{r}} \mathcal{N}(\mathbf{n})$. Therefore

$$
\Gamma(U, \widetilde{N})=\Gamma\left(Z, h_{*} \widetilde{N}\right)=\Gamma\left(Z, \bigoplus_{\mathbf{n} \in \mathbb{Z}^{r}} \mathcal{N}(\mathbf{n})\right)=\bigoplus_{\mathbf{n} \in \mathbb{Z}^{r}} \Gamma(Z, \mathcal{N}(\mathbf{n})) .
$$


It follows, in particular, that $\Gamma(U, \widetilde{N})$ is an $r$-graded $T$-module. Set $\Phi(N)=$ $\bigoplus_{\mathbf{n} \in \mathbb{Z}^{r}} \mathcal{N}(\mathbf{n})$. This defines an exact functor $\Phi$ from the category of $r$-graded $T$-modules to the category of quasi-coherent $\mathcal{O}_{Z}$-modules. So there is an exact sequence

$$
0 \rightarrow \underline{H}_{T^{+}}^{0}\left(I^{\bullet}\right) \rightarrow I^{\bullet} \rightarrow \Gamma\left(Z, \Phi\left(I^{\bullet}\right)\right) \rightarrow 0
$$

of complexes of $r$-graded $T$-modules. Observe that $\Phi\left(I^{\bullet}\right)$ is a complex of injective $\mathcal{O}_{Z}$-modules. Indeed, if $\mathcal{I}^{p}$ is the quasi-coherent $\mathcal{O}_{Z}$-module corresponding to $I^{p}$ $(p \in \mathbb{N})$, we know from Lemma 1.3 that $\mathcal{I}^{p}$ is injective. Then $\mathcal{I}^{p}(\mathbf{n})$ is injective for all $\mathbf{n} \in \mathbb{Z}^{r}$ so that also $\Phi\left(I^{p}\right)=\bigoplus_{\mathbf{n} \in \mathbb{Z}^{r}} \mathcal{I}^{p}(\mathbf{n})$ is injective. In the derived category of the category of $r$-graded $T$-modules the above exact sequence then gives an exact triangle

$$
\mathbf{R} \underline{H}_{T^{+}}^{0}(M) \rightarrow M \rightarrow \mathbf{R} \Gamma(Z, \Phi(M)) \rightarrow \mathbf{R} \underline{H}_{T^{+}}^{0}(M)[1] .
$$

Since $H_{\mathfrak{a}}^{0} \underline{H}_{T^{+}}^{0}=\underline{H}_{\left(\mathfrak{a}, T^{+}\right)}^{0}$ and $H_{\mathfrak{a}}^{0} \Gamma(Z, \cdot)=\Gamma_{E}(Z, \cdot)$, we obtain the desired sequence by applying the functor $\mathbf{R} H_{\mathfrak{a}}^{0}$ and taking cohomology.

In order to prove the second claim we first note that by setting $\Psi(N)=$ $\bigoplus_{\mathbf{n} \in \mathbb{Z}^{r}} \mathcal{N}_{n_{j}}(\hat{\mathbf{n}})$ for every $r$-graded $T$-module $N$, we obtain an exact functor $\Psi$ from the category of $r$-graded $T$-modules to the category of quasi-coherent $\mathcal{O}_{Y}$-modules. The module $\Gamma(Y, \Psi(N))$ has an obvious structure of an $r$-graded $T$-module. Moreover, the canonical homomorphisms $\mathcal{N}_{n_{j}}(\hat{\mathbf{n}}) \rightarrow g_{*}(\mathcal{N}(\mathbf{n}))$ induce a morphism of functors $\eta: \Psi \rightarrow g_{*} \Phi$ such that there is a commutative diagram

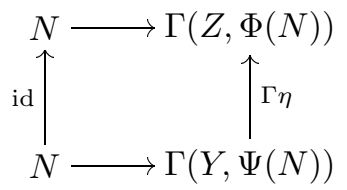

of $r$-graded $T$-modules.

Now observe that by writing $M=\bigoplus_{k \in \mathbb{Z}} M_{\ldots, k, \ldots}$ and $I^{\bullet}=\bigoplus_{k \in \mathbb{Z}} I_{\ldots, k, \ldots}^{\bullet}$, we can consider $0 \rightarrow M \rightarrow I^{\bullet}$ as an $H_{S^{+}}^{0}$-acyclic resolution of $M$ in the category of $r-1$-graded $S$-modules. Moreover, by using the Sancho de Salas sequence, we see that $H_{F}^{i}\left(Y, \Psi\left(I^{p}\right)\right)=0$ for $i>0$ and $p \in \mathbb{N}$. When $\mathfrak{a}=0$, this gives that also $H^{i}\left(Y, \Psi\left(I^{p}\right)\right)=0$ for $i>0$ and $p \in \mathbb{N}$.

It follows that there is a commutative diagram

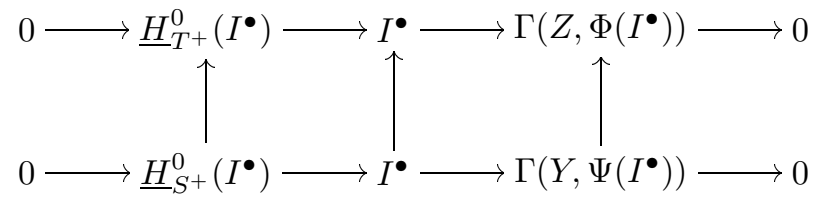

of complexes of $r$-graded $T$-modules. This means that in the derived category we get a morphism of triangles

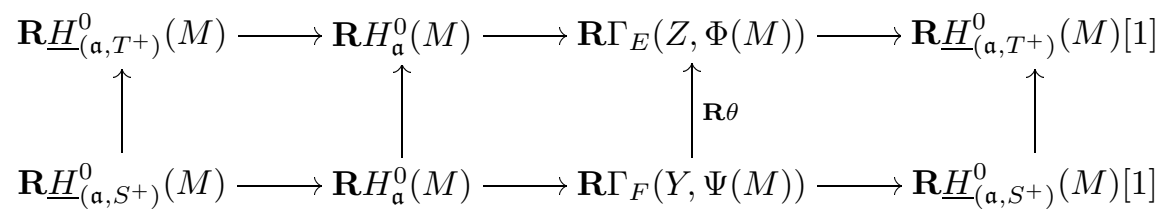


where $\Gamma_{F}=\Gamma_{F}(Y, \cdot), \Gamma_{E}=\Gamma_{E}(Z, \cdot)=\Gamma_{F} g_{*}$ and

$$
\mathbf{R} \theta:\left(\mathbf{R} \Gamma_{F}\right) \Psi=\mathbf{R}\left(\Gamma_{F} \Psi\right) \rightarrow \mathbf{R}\left(\Gamma_{E} \Phi\right)=\left(\mathbf{R} \Gamma_{E}\right) \Phi
$$

is the morphism of functors induced by $\theta=\Gamma_{F} \eta$. By taking the cohomology we obtain a diagram of desired type. It remains to check that the resulting homomorphisms $H^{i}(\mathbf{R} \theta): H_{F}^{i}(Y, \Psi(M)) \rightarrow H_{E}^{i}(Z, \Phi(M))$ factor in the right way.

Consider the commutative diagram

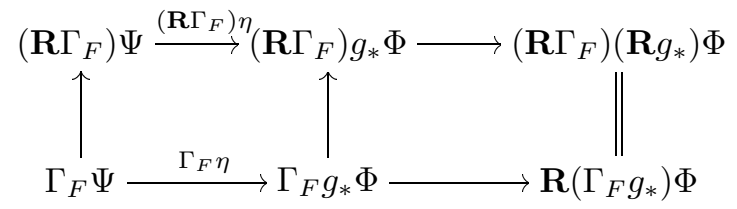

where $\Gamma_{F} \Psi \rightarrow\left(\mathbf{R} \Gamma_{F}\right) \Psi=\mathbf{R}\left(\Gamma_{F} \Psi\right)$ and $\Gamma_{F} g_{*} \Phi \rightarrow \mathbf{R}\left(\Gamma_{F} g_{*}\right) \Phi=\mathbf{R}\left(\Gamma_{F} g_{*} \Phi\right)$ are canonical morphisms of functors. As $\mathbf{R} \theta$ is the unique morphism of functors $\left(\mathbf{R} \Gamma_{F}\right) \Psi \rightarrow \mathbf{R}\left(\Gamma_{F} g_{*}\right) \Phi$ making the above diagram commutative, we see that it is equal to the composition

$$
\left(\mathbf{R} \Gamma_{F}\right) \Psi \rightarrow\left(\mathbf{R} \Gamma_{F}\right) g_{*} \Phi \rightarrow\left(\mathbf{R} \Gamma_{F}\right)\left(\mathbf{R} g_{*}\right) \Phi=\mathbf{R}\left(\Gamma_{F} g_{*}\right) \Phi
$$

The cohomology maps corresponding to $\left(\mathbf{R} \Gamma_{F}\right) g_{*} \Phi(M) \rightarrow\left(\mathbf{R} \Gamma_{F}\right)\left(\mathbf{R} g_{*}\right) \Phi(M)$ are edge homomorphisms of the Grothendieck spectral sequence of the composite functor $\Gamma_{F} g_{*}$. This implies the claim, since these are well-known to be the homomorphisms $H_{E}^{i}\left(Y, g_{*} \Phi(M)\right) \rightarrow H_{E}^{i}(Z, \Phi(M))$.

By choosing $\mathfrak{a}=0$ we immediately obtain

1.5. Corollary. Let $T$ be a standard $r$-graded ring and let $M$ be an $r$-graded $T$ module. With the preceding notation, we have an exact sequence

$$
0 \rightarrow \underline{H}_{T^{+}}^{0}(M) \rightarrow M \rightarrow \bigoplus_{\mathbf{n} \in \mathbb{Z}^{r}} \Gamma(Z, \mathcal{M}(\mathbf{n})) \rightarrow \underline{H}_{T^{+}}^{1}(M) \rightarrow 0
$$

and isomorphisms

$$
\underline{H}_{T^{+}}^{i}(M)=\bigoplus_{\mathbf{n} \in \mathbb{Z}^{r}} H^{i-1}(Z, \mathcal{M}(\mathbf{n})) \quad(i>1) .
$$

We next note that the theorem of Serre about vanishing of sheaf cohomology has the following multigraded analogue (cf. [12, Lemma (4.2)]):

1.6. Theorem. Let $T$ be a standard $r$-graded ring and let $M$ be a finitely generated $r$-graded T-module. Let the notation be as above. If $n_{1}, \ldots, n_{r} \gg 0$, we have $\Gamma(Z, \mathcal{M}(\mathbf{n}))=M_{\mathbf{n}}$ and $H^{i}(Z, \mathcal{M}(\mathbf{n}))=0$ for $i>0$.

Let $A$ be a ring and let $I_{1}, \ldots, I_{r} \subset A$ be ideals. The multi-Rees algebra $R_{A}\left(I_{1}, \ldots, I_{r}\right)$ is the $r$-graded ring

$$
R_{A}\left(I_{1}, \ldots, I_{r}\right)=\bigoplus_{\mathbf{n} \in \mathbb{N}^{r}} I_{1}^{n_{1}} \cdots I_{r}^{n_{r}}
$$

We often identify $R_{A}\left(I_{1}, \ldots, I_{r}\right)$ with the subring $A\left[I_{1} t_{1}, \ldots, I_{r} t_{r}\right]$ of $A\left[t_{1}, \ldots, t_{r}\right]$. Note that $R_{A}\left(I_{1}, \ldots, I_{r}\right)=R_{S}\left(I_{j} S\right)$ where $S=R_{A}\left(I_{1}, \ldots, I_{j-1}, I_{j+1}, \ldots, I_{r}\right)$. It follows, in particular, that the scheme $Z=\operatorname{Proj} R_{A}\left(I_{1}, \ldots, I_{r}\right)$ can be considered as 
the blow-up of the scheme $Y=\operatorname{Proj} R_{A}\left(I_{1}, \ldots, I_{j-1}, I_{j+1}, \ldots, I_{r}\right)$ along the closed subscheme defined by the sheaf of ideals $I_{j} \mathcal{O}_{Y}$. The multi-form ring

$$
g r_{A}\left(I_{1}, \ldots, I_{r}\right)=R_{A}\left(I_{1}, \ldots, I_{r}\right) /\left(I_{1} \cdots I_{r}\right) R_{A}\left(I_{1}, \ldots, I_{r}\right) .
$$

The corresponding diagonal subrings are $\left(R_{A}\left(I_{1}, \ldots, I_{r}\right)\right)^{\Delta}=R_{A}\left(I_{1} \cdots I_{r}\right)$ and $\left(g r_{A}\left(I_{1}, \ldots, I_{r}\right)\right)^{\Delta}=g r_{A}\left(I_{1} \cdots I_{r}\right)$. If ht $I_{j}>0(j=1, \ldots, r)$, it follows easily from [1, Theorem 4.4.6] that $\operatorname{dim} R_{A}\left(I_{1}, \ldots, I_{r}\right)=\operatorname{dim} A+r$. If, moreover, $A$ is local, $\operatorname{dim} g r_{A}\left(I_{1}, \ldots, I_{r}\right)=\operatorname{dim} A+r-1$.

\section{MAin RESUlts}

In this section we want to give necessary and sufficient conditions for the CohenMacaulayness of a standard bigraded ring $T=\bigoplus_{p, q \geq 0} T_{p, q}$ defined over a local ring $(A, \mathfrak{m})$. Before proving our main Theorem 2.5 we are going to state several lemmata.

In the proof of Theorem 2.5 we shall make use of the multigraded Sancho de Salas sequence (Theorem 1.4). Working with sheaf cohomology on the schemes $\operatorname{Proj} T$ and Proj $T_{, 0}, \operatorname{Proj} T_{0}$, respectively, we need the following general fact about vanishing of the higher direct-image sheaves.

2.1. Lemma. Let $f: X \rightarrow Y$ be a proper morphism of schemes of finite type over a ring $A$. Let $\mathcal{F}$ be a coherent sheaf on $X$. Let $\mathcal{L}$ be an ample invertible sheaf on $Y$. Then $R^{i} f_{*} \mathcal{F}=0$ for all $i>0$ if and only if $H^{i}\left(X, \mathcal{F} \otimes f^{*} \mathcal{L}^{\otimes n}\right)=0$ for all $i>0$ when $n \gg 0$.

Proof. Consider the Leray spectral sequence

$$
E_{2}^{p, q}=H^{p}\left(Y, R^{q} f_{*}\left(\mathcal{F} \otimes f^{*} \mathcal{L}^{\otimes n}\right)\right) \Rightarrow H^{p+q}\left(X, \mathcal{F} \otimes f^{*} \mathcal{L}^{\otimes n}\right) .
$$

Note that by the projection formula $R^{q} f_{*}\left(\mathcal{F} \otimes f^{*} \mathcal{L}^{\otimes n}\right)=R^{q} f_{*}(\mathcal{F}) \otimes \mathcal{L}^{\otimes n}$.

Suppose first that there exists $n_{0} \in \mathbb{N}$ such that $H^{i}\left(X, \mathcal{F} \otimes f^{*} \mathcal{L}^{\otimes n}\right)=0$ for all $i>0$ when $n>n_{0}$. Let $i>0$. By the theorem of Serre we can choose $n_{1} \in \mathbb{N}$ such that $H^{p}\left(Y, R^{q} f_{*}(\mathcal{F}) \otimes \mathcal{L}^{\otimes n}\right)=0$ for all $p>0$ and $q<i$ when $n>n_{1}$. The Leray spectral sequence therefore gives

$$
\Gamma\left(Y, R^{i} f_{*}(\mathcal{F}) \otimes \mathcal{L}^{\otimes n}\right)=H^{i}\left(X, \mathcal{F} \otimes f^{*} \mathcal{L}^{\otimes n}\right)=0
$$

for $n>\max \left(n_{0}, n_{1}\right)$. This implies that $R^{i} f_{*} \mathcal{F}=0$ as wanted. Conversely, if $R^{i} f_{*} \mathcal{F}=0$ for all $i>0$, the Leray spectral sequence degenerates. By using the theorem of Serre again, we can then find $n_{0} \in \mathbb{N}$ such that

$$
H^{i}\left(X, \mathcal{F} \otimes f^{*} \mathcal{L}^{\otimes n}\right)=H^{i}\left(Y, f_{*}(\mathcal{F}) \otimes \mathcal{L}^{\otimes n}\right)=0
$$

for all $i>0$ when $n>n_{0}$.

We also need to consider the local cohomology with respect to the ideal $\mathfrak{M}^{+}=$ $\left(\mathfrak{m}, T^{+}\right) \subset T$. Observe that $\mathfrak{M}^{+}=\mathfrak{M}^{\Delta} T$ where $\mathfrak{M}$ is the homogeneous maximal ideal of $T$.

2.2. Lemma. Let $T$ be a bigraded ring defined over a ring $A$. Let $\mathfrak{A} \subset T^{\Delta}$ be a homogeneous ideal. Let $M$ be a bigraded $T$-module. We then have for all $p, q \in \mathbb{Z}$ and $i \geq 0$ that

$$
\left[\underline{H}_{\mathfrak{A} T}^{i}(M)\right]_{p, q}= \begin{cases}{\left[\underline{H}_{\mathfrak{A}}^{i}\left((M(p-q, 0))^{\Delta}\right)\right]_{q}} & \text { if } p \geq q, \\ {\left[\underline{H}_{\mathfrak{A}}^{i}\left((M(0, q-p))^{\Delta}\right)\right]_{p}} & \text { if } p<q .\end{cases}
$$


Proof. Consider $T^{\Delta}$ as a bigraded subring of $T$ concentrated in degrees $(p, p)$ $(p \in \mathbb{Z})$. Then $M$ is a bigraded $T^{\Delta}$-module and $\underline{H}_{\mathfrak{A} T}^{i}(M)=\underline{H}_{\mathfrak{A}}^{i}(M)$. Set $N_{k}=(M(k, 0))^{\Delta}(k \in \mathbb{Z})$. We can now write

$$
M=\bigoplus_{k \in \mathbb{Z}}\left(\bigoplus_{q \in \mathbb{Z}} M_{q+k, q}\right)=\bigoplus_{k \in \mathbb{Z}} N_{k}(-k, 0)
$$

where $N_{k}$ is considered as bigraded $T^{\Delta}$-module with $\left[N_{k}\right]_{p, q}=0$ if $p \neq q$. Then

$$
\left[\underline{H}_{\mathfrak{A}}^{i}(M)\right]_{p, q}=\bigoplus_{k \in \mathbb{Z}}\left[\underline{H}_{\mathfrak{A}}^{i}\left(N_{k}\right)\right]_{p-k, q}=\left[\underline{H}_{\mathfrak{A}}^{i}\left(N_{p-q}\right)\right]_{q} .
$$

This implies the claim, because $M(k, 0)=M(0-k)(k, k)$ for all $k \in \mathbb{Z}$.

2.3. Lemma. Let $R$ be a graded ring defined over a local ring $(A, \mathfrak{m})$. Let $\mathfrak{M}$ be the homogeneous maximal ideal of $R$. Let $\mathfrak{a} \subset \mathfrak{m}$ be an ideal. Let $M$ be a finitely generated graded $R$-module and $n_{0} \in \mathbb{Z}$. Then $\left[\underline{H}_{\mathfrak{M}}^{i}(M)\right]_{n}=0$ for all $n \geq n_{0}$ and $i \geq 0$ if and only if $\left[\underline{H}_{\left(\mathfrak{a}, R^{+}\right)}^{i}(M)\right]_{n}=0$ for all $n \geq n_{0}$ and $i \geq 0$.

Proof. Given an ideal $\mathfrak{b} \supset \mathfrak{a}$, it is immediately clear from the spectral sequence

$$
E_{2}^{i, j}=H_{\mathfrak{b}}^{i}\left(\left[\underline{H}_{\left(\mathfrak{a}, R^{+}\right)}^{j}(M)\right]_{n}\right) \Rightarrow\left[\underline{H}_{\left(\mathfrak{b}, R^{+}\right)}^{i+j}(M)\right]_{n}
$$

that if $\left[\underline{H}_{\left(\mathfrak{a}, R^{+}\right)}^{i}(M)\right]_{n}=0$ for all $i \geq 0$, also $\left[\underline{H}_{\left(\mathfrak{b}, R^{+}\right)}^{i}(M)\right]_{n}=0$ for all $i \geq 0$. It is therefore enough to prove that $\left[\underline{H}_{\mathfrak{M}}^{i}(M)\right]_{n}=0$ for all $n \geq n_{0}$ and $i \geq 0$ implies $\left[\underline{H}_{R^{+}}^{i}(M)\right]_{n}=0$ for all $n \geq n_{0}$ and $i \geq 0$.

By moving to the completion $\widehat{R}=R \otimes_{A} \widehat{A}$, we can assume that $A$ is complete. For any $\mathfrak{p} \in \operatorname{Spec} A$, set $\mathfrak{P}=\mathfrak{p} A_{\mathfrak{p}} \oplus R_{\mathfrak{p}}^{+}$. Let us first show that $\left[\underline{H}_{\mathfrak{P}}^{i}\left(M_{\mathfrak{p}}\right)\right]_{n}=0$ for all $\mathfrak{p} \in \operatorname{Spec} A, n \geq n_{0}$ and $i \geq 0$. Express $R$ as a quotient of some polynomial ring $S$ defined over a local Gorenstein ring $B$. According to the graded version of local duality we have for all $i \geq 0$

$$
\underline{H}_{\mathfrak{M}}^{i}(M)=\underline{\operatorname{Hom}}_{S}\left(\underline{\operatorname{Ext}}_{S}^{\operatorname{dim} S-i}(M, S(a)), \underline{E}_{S}(k)\right)
$$

where $a \in \mathbb{Z}$ and $\underline{E}_{S}(k)$ is the graded injective envelope of the residue field $k$ of $B$. Since $\underline{E}_{S}(k)=\underline{\operatorname{Hom}}_{B}\left(S, E_{B}(k)\right)$, this gives

$$
\left[\underline{H}_{\mathfrak{M}}^{i}(M)\right]_{n}=\operatorname{Hom}_{B}\left(\left[\underline{\operatorname{Ext}}_{S}^{\operatorname{dim}} S-i(M, S(a))\right]_{-n}, E_{B}(k)\right)
$$

for all $n \in \mathbb{Z}$. The assumption thus implies that $\left[\operatorname{Ext}_{S}^{i}(M, S(a))\right]_{-n}=0$ for all $n \geq n_{0}$ and $i \geq 0$. Let $\mathfrak{p} \in \operatorname{Spec} A$. Suppose that $A=B / I$ where $I \subset B$ is an ideal. Write $\mathfrak{p}=\mathfrak{q} / I$ for some $\mathfrak{q} \in \operatorname{Spec} B, \mathfrak{q} \subset I$. Then

$$
\left(\left[\underline{\operatorname{Ext}}_{S}^{i}(M, S(a))\right]_{-n}\right)_{\mathfrak{q}}=\left[\underline{\operatorname{Ext}}_{S_{\mathfrak{q}}}^{i}\left(M_{\mathfrak{p}}, S_{\mathfrak{q}}(a)\right)\right]_{-n} .
$$

By using the local duality again, we then get that $\left[\underline{H}_{\mathfrak{P}}^{i}\left(M_{\mathfrak{p}}\right)\right]_{n}=0$ for all $n \geq n_{0}$ and $i \geq 0$ as desired.

Let us now use induction on $\operatorname{dim} A$ to prove the actual claim. If $\operatorname{dim} A=0$, $\operatorname{Rad}\left(R^{+}\right)=\mathfrak{M}$ and there is nothing to prove. Suppose $\operatorname{dim} A>0$. If $\mathfrak{p} \in \operatorname{Spec} A$, $\mathfrak{p} \neq \mathfrak{m}$, we saw above that $\left[\underline{H}_{\mathfrak{P}}^{i}\left(M_{\mathfrak{p}}\right)\right]_{n}=0$ for all $n \geq n_{0}$ and $i \geq 0$. The induction hypothesis then gives that

$$
\left[\underline{H}_{R_{\mathfrak{p}}^{+}}^{i}\left(M_{\mathfrak{p}}\right)\right]_{n}=\left(\left[\underline{H}_{R^{+}}^{i}(M)\right]_{n}\right)_{\mathfrak{p}}=0 .
$$


As $\left[\underline{H}_{R^{+}}^{i}(M)\right]_{n}$ is a finitely generated $A$-module, this means that it must have finite length. But then the spectral sequence

$$
E_{2}^{i, j}=H_{\mathfrak{m}}^{i}\left(\left[\underline{H}_{R^{+}}^{j}(M)\right]_{n}\right) \Rightarrow\left[\underline{H}_{\mathfrak{M}}^{i+j}(M)\right]_{n}
$$

degenerates to give $\left[\underline{H}_{R^{+}}^{i}(M)\right]_{n}=\left[\underline{H}_{\mathfrak{M}}^{i}(M)\right]_{n}=0$ for all $n \geq n_{0}$ and $i \geq 0$.

2.4. Lemma. Let $T$ be a bigraded ring defined over a local ring $(A, \mathfrak{m})$. Let $\mathfrak{M}$ be the homogeneous maximal ideal of $T$. Set $\mathfrak{M}^{+}=\left(\mathfrak{m}, T^{+}\right) \subset T$. Let $M$ be a finitely generated bigraded $T$-module. Then $\left[\underline{H}_{\mathfrak{M}^{+}}^{i}(M)\right]_{p, q}=0$ for all $p, q \geq 0$ and $i \geq 0$ if and only if $\left[\underline{H}_{T^{+}}^{i}(M)\right]_{p, q}=0$ for all $p, q \geq 0$ and $i \geq 0$.

Proof. Set $Q=T^{\Delta}, \mathfrak{N}=\mathfrak{M}^{\Delta}$ and let $N_{k}=(M(k, 0))^{\Delta}(k \in \mathbb{Z})$. According to Lemma 2.2 we have $\left[\underline{H}_{\mathfrak{M}^{+}}^{i}(M)\right]_{p, q}=\left[\underline{H}_{\mathfrak{M}}^{i}\left(N_{p-q}\right)\right]_{q}$. It follows that $\left[\underline{H}_{\mathfrak{M}^{+}}^{i}(M)\right]_{p, q}=$ 0 for $p, q \geq 0$ and $i \geq 0$ is equivalent to $\left[\underline{H}_{\mathfrak{N}}^{i}\left(N_{k}\right)\right]_{q}=0$ for $k \in \mathbb{Z}, q \geq \max (0,-k)$ and $i \geq 0$. By Lemma 2.3 this is further equivalent to $\left[\underline{H}_{Q^{+}}^{i}\left(N_{k}\right)\right]_{q}=0$ for $k \in$ $\mathbb{Z}, q \geq \max (0,-k)$ and $i \geq 0$. By using Lemma 2.2 again we see that this is the same as $\left[\underline{H}_{T^{+}}^{i}(M)\right]_{p, q}=0$ for $p, q \geq 0$ and $i \geq 0$.

Let $T$ be an $\mathbb{N}^{r}$-graded ring defined over a local ring and let $\mathfrak{M}$ be the homogeneous maximal ideal of $T$. Define for all $j=1, \ldots, r$ the $a$-invariants

$$
a^{j}(T)=\sup \left\{k \in \mathbb{Z} \mid\left[\underline{H}_{\mathfrak{M}}^{\operatorname{dim} T}(T)\right]_{\mathbf{n}} \neq 0 \text { for some } \mathbf{n} \in \mathbb{Z}^{r} \text { with } n_{j}=k\right\} .
$$

Note that by Lemma $1.1 a^{j}(T)$ coincides with the usual $a$-invariant $a\left(T_{\mathfrak{N}}\right)$ of the graded ring $T_{\mathfrak{N}}$ where $\mathfrak{N}$ is the homogeneous maximal ideal of the $\operatorname{ring} T_{\ldots, 0, \ldots}{ }^{(j)}$.

We are now ready to prove

2.5. Theorem. Let $T$ be a standard bigraded ring of dimension $d+2$ defined over a local ring $(A, \mathfrak{m})$. Suppose that $\operatorname{dim} T_{0, .}, \operatorname{dim} T_{, 0}<d+2$ and $a^{1}(T), a^{2}(T)<0$. Let $\mathfrak{M}$ be the homogeneous maximal ideal of $T$ and $\mathfrak{M}^{+}=\left(\mathfrak{m}, T^{+}\right) \subset T$. Set $Z=\operatorname{Proj} T$ and $E=V_{+}(\mathfrak{m} T) \subset Z$. Then the following conditions are equivalent:

1) $T$ is Cohen-Macaulay;

2) a) $\left[\underline{H}_{\mathfrak{M}^{+}}^{i}(T)\right]_{p, q}=0$ if $p, q \geq 0$ or $p, q<0$ and $i<d+1$,

b) $\left[\underline{H}_{\mathfrak{M}_{+}}^{d+1}(T)\right]_{p, q}=0$ if $p, q \geq 0$;

3) a) $\left[\underline{H}_{\mathfrak{M} \Delta}^{i}\left((T(p, 0))^{\Delta}\right)\right]_{q}=0$ if $p \geq 0, q \notin\{-p, \ldots,-1\}$ and $i<d+1$,

b) $\left[\underline{H}_{\mathfrak{M} \Delta}^{i}\left((T(0, q))^{\Delta}\right)\right]_{p}=0$ if $q \geq 0, p \notin\{-q, \ldots,-1\}$ and $i<d+1$,

c) $\left[\underline{H}_{\mathfrak{M} \Delta}^{d+1}\left((T(p, 0))^{\Delta}\right)\right]_{q}=0,\left[\underline{H}_{\mathfrak{M} \Delta}^{d+1}\left((T(0, q))^{\Delta}\right)\right]_{p}=0$ if $p, q \geq 0$;

4) a) The homomorphism $T_{p, q} \rightarrow \Gamma\left(Z, \mathcal{O}_{Z}(p, q)\right)$ is an isomorphism for $p, q \geq 0$,

b) $H^{i}\left(Z, \mathcal{O}_{Z}(p, q)\right)=0$ if $p, q \geq 0$ and $i>0$,

c) $H_{E}^{i}\left(Z, \mathcal{O}_{Z}(p, q)\right)=0$ if $p, q<0$ and $i<d$.

It follows, in particular, that if $T$ is Cohen-Macaulay, then so is $T^{\Delta}$.

Proof.

1) $\Leftrightarrow 2)$ Set $\mathfrak{M}_{1}^{+}=\left(\mathfrak{m}, T_{1}^{+}\right)$and $\mathfrak{M}_{2}^{+}=\left(\mathfrak{m}, T_{2}^{+}\right)$. Then $\mathfrak{M}_{1}^{+}+\mathfrak{M}_{2}^{+}=\mathfrak{M}$ and $\mathfrak{M}_{1}^{+} \cap \mathfrak{M}_{2}^{+}=\mathfrak{M}^{+}$. Consider the Mayer-Vietoris sequence of local cohomology

$$
\cdots \rightarrow \underline{H}_{\mathfrak{M}}^{i}(T) \rightarrow \underline{H}_{\mathfrak{M}_{1}^{+}}^{i}(T) \oplus \underline{H}_{\mathfrak{M}_{2}^{+}}^{i}(T) \rightarrow \underline{H}_{\mathfrak{M}^{+}}^{i}(T) \rightarrow \underline{H}_{\mathfrak{M}}^{i+1}(T) \rightarrow \cdots
$$

This implies that $\underline{H}_{\mathfrak{M}}^{i}(T)=0$ for $i<d+2$ if and only if the homomorphism

$$
\underline{H}_{\mathfrak{M}_{1}^{+}}^{i}(T) \oplus \underline{H}_{\mathfrak{M}_{2}^{+}}^{i}(T) \rightarrow \underline{H}_{\mathfrak{M}^{+}}^{i}(T)
$$


is an isomorphism for $i<d+1$ and injective for $i=d+1$. We now have $\mathfrak{M}_{1}^{+}=\mathfrak{M}_{, 0} T$ and $\mathfrak{M}_{2}^{+}=\mathfrak{M}_{0,}, T$. Since $T=\bigoplus_{q \geq 0} T, q=\bigoplus_{p \geq 0} T_{p, .}$, it follows that

$$
\left[\underline{H}_{\mathfrak{M}_{1}^{+}}^{i}(T)\right]_{p, q}=\left[\underline{H}_{\mathfrak{M}_{,, 0}}^{i}(T \cdot, q)\right]_{p} \quad \text { and } \quad\left[\underline{H}_{\mathfrak{M}_{2}^{+}}^{i}(T)\right]_{p, q}=\left[\underline{H}_{\mathfrak{M}_{0, .}}^{i}\left(T_{p, \cdot}\right)\right]_{q}
$$

for all $p, q \in \mathbb{Z}$ and $i \geq 0$. Therefore we obtain for all $i \geq 0$ that

$$
\left[\underline{H}_{\mathfrak{M}_{1}^{+}}^{i}(T)\right]_{p, q}=0 \text { if } q<0 \text { and }\left[\underline{H}_{\mathfrak{M}_{2}^{+}}^{i}(T)\right]_{p, q}=0 \text { if } p<0 .
$$

Since $\operatorname{dim} T_{0, \cdot}, \operatorname{dim} T_{,, 0} \leq d+1$, also $\underline{H}_{\mathfrak{M}_{1}^{+}}^{i}(T)=0$ and $\underline{H}_{\mathfrak{M}_{2}^{+}}^{i}(T)=0$ if $i>d+1$.

Suppose now that $T$ is Cohen-Macaulay. As we also have $a^{1}(T), a^{2}(T)<0$, it follows that $\left[\underline{H}_{\mathfrak{M}}^{i}(T)\right]_{p, q}=0$ for all $p \geq 0$ or $q \geq 0$ and $i \geq 0$. By first localizing at the homogeneous maximal ideals of $T_{0, \text {. and }} T_{\cdot, 0}$ respectively, and then using Lemma 1.1, we may apply Lemma 2.3 to get

$$
\left[\underline{H}_{\mathfrak{M}_{1}^{+}}^{i}(T)\right]_{p, q}=0 \quad \text { and } \quad\left[\underline{H}_{\mathfrak{M}_{2}^{+}}^{i}(T)\right]_{p, q}=0
$$

for all $p, q \geq 0$ and $i \geq 0$. The remarks made above then imply that 2) holds.

Suppose then that 2) holds. So $\left[\underline{H}_{\mathfrak{M}^{+}}^{i}(T)\right]_{p, q}=0$ for all $p, q \geq 0$ and $i \geq 0$. We can now use Lemma 2.4 to get $\left[\underline{H}_{T^{+}}^{i}(T)\right]_{p, q}=0$ for all $p, q \geq 0$ and $i \geq 0$. This implies by Corollary 1.5 that the homomorphism $T_{p, q} \rightarrow \Gamma\left(Z, \mathcal{O}_{Z}(p, q)\right)$ is an isomorphism and $H^{i}\left(Z, \mathcal{O}_{Z}(p, q)\right)=0$ for all $p, q \geq 0$ and $i>0$. Set $Y=$ $\operatorname{Proj} T_{\cdot, 0}, F=V_{+}\left(\mathfrak{m} T_{\cdot, 0}\right) \subset Y$ and let $g: Z \rightarrow Y$ be the canonical projection. If $p \in \mathbb{Z}$ and $q \geq 0$, we now have

$$
H^{i}\left(Z, \mathcal{O}_{Z}(p, q) \otimes g^{*} \mathcal{O}_{Y}\left(p^{\prime}\right)\right)=H^{i}\left(Z, \mathcal{O}_{Z}\left(p+p^{\prime}, q\right)\right)=0
$$

for $p^{\prime} \geq-p$ and $i>0$. We can then use Lemma 2.1 to obtain $R^{i} g_{*}\left(\mathcal{O}_{Z}(p, q)\right)=0$ for $i>0$. This implies that the Leray spectral sequence

$$
E_{2}^{i, j}=H_{F}^{i}\left(Y, R^{j} g_{*}\left(\mathcal{O}_{Z}(p, q)\right)\right) \Rightarrow H_{E}^{i+j}\left(Z, \mathcal{O}_{Z}(p, q)\right)
$$

degenerates. The edge homomorphisms $H_{F}^{i}\left(Y, g_{*}\left(\mathcal{O}_{Z}(p, q)\right)\right) \rightarrow H_{E}^{i}\left(Z, \mathcal{O}_{Z}(p, q)\right)$ are thus isomorphisms for all $p \in \mathbb{Z}, q \geq 0$ and $i \geq 0$. Let $\mathcal{T}_{q}$ denote the quasicoherent $\mathcal{O}_{Y}$-module corresponding to $T_{\cdot, q}(q \geq 0)$. Also the canonical homomorphism $\mathcal{T}_{q}(p) \rightarrow g_{*}\left(\mathcal{O}_{Z}(p, q)\right)$ is now an isomorphism for $p \in \mathbb{Z}$ and $q \geq 0$. As $g_{*}\left(\mathcal{O}_{Z}(p, q)\right) \otimes \mathcal{O}_{Y}\left(p^{\prime}\right)=g_{*}\left(\mathcal{O}_{Z}\left(p+p^{\prime}, q\right)\right)$, we can see this by showing that $\Gamma\left(Y, \mathcal{T}_{q}(p)\right) \rightarrow \Gamma\left(Z, \mathcal{O}_{Z}(p, q)\right)$ is an isomorphism for $p \gg 0$. But for $p \gg 0$, $\Gamma\left(Y, \mathcal{T}_{q}(p)\right)=T_{p, q}=\Gamma\left(Z, \mathcal{O}_{Z}(p, q)\right)$. It follows that the homomorphisms

$$
H_{F}^{i}\left(Y, \mathcal{T}_{q}(p)\right) \rightarrow H_{F}^{i}\left(Y, g_{*}\left(\mathcal{O}_{Z}(p, q)\right)\right) \rightarrow H_{E}^{i}\left(Z, \mathcal{O}_{Z}(p, q)\right)
$$

mentioned in Theorem 1.4 are isomorphisms for all $p \in \mathbb{Z}, q \geq 0$ and $i \geq 0$. Combined with the five lemma Theorem 1.4 then implies that the homomorphism

$$
\left[\underline{H}_{\mathfrak{M}_{1}^{+}}^{i}(T)\right]_{p, q} \rightarrow\left[\underline{H}_{\mathfrak{M}^{+}}^{i}(T)\right]_{p, q}
$$

is an isomorphism for all $p \in \mathbb{Z}, q \geq 0$ and $i \geq 0$. Similarly, the homomorphism

$$
\left[\underline{H}_{\mathfrak{M}_{2}^{+}}^{i}(T)\right]_{p, q} \rightarrow\left[\underline{H}_{\mathfrak{M}^{+}}^{i}(T)\right]_{p, q}
$$

is an isomorphism for all $p \geq 0, q \in \mathbb{Z}$ and $i \geq 0$. For $p, q \geq 0$, this means that

$$
\left[\underline{H}_{\mathfrak{M}_{1}^{+}}^{i}(T)\right]_{p, q}=0 \quad \text { and } \quad\left[\underline{H}_{\mathfrak{M}_{2}^{+}}^{i}(T)\right]_{p, q}=0 .
$$

By taking into account the formulas $(\dagger)$, it now follows that if $p \geq 0$ or $q \geq 0$, the homomorphism $(*)$ is an isomorphism in degree $(p, q)$ for all $i \geq 0$. By assumption $\left[\underline{H}_{\mathfrak{M}^{+}}^{i}(T)\right]_{p, q}=0$ for $p, q<0$ and $i<d+1$. We then see that in degrees $(p, q)$ with 
$p, q<0(*)$ is an isomorphism for $i<d+1$ and injective for $i=d+1$. This means that $T$ is Cohen-Macaulay as wanted.

$2) \Leftrightarrow 3$ ) This is an immediate consequence of Lemma 2.2.

$2) \Leftrightarrow 4)$ Corollary 1.5 implies that 4) a) and b) are equivalent to $\left[\underline{H}_{T^{+}}^{i}(T)\right]_{p, q}=0$ for all $p, q \geq 0$ and $i \geq 0$. By Lemma 2.4 this is in turn equivalent to $\left[\underline{H}_{\mathfrak{M}^{+}}^{i}(T)\right]_{p, q}=$ 0 for all $p, q \geq 0$ and $i \geq 0$. By using Theorem 1.4 we get that $H_{E}^{i}\left(Z, \mathcal{O}_{Z}(p, q)\right)=$ $\left[\underline{H}_{\mathfrak{M}^{+}}^{i+1}(T)\right]_{p, q}$ for all $p, q<0$ and $i \geq 0$. Therefore $\left[\underline{H}_{\mathfrak{M}^{+}}^{i}(T)\right]_{p, q}=0$ for $p, q<0$ and $i<d+1$ is equivalent to $H_{E}^{i}\left(Z, \mathcal{O}_{Z}(p, q)\right)=0$ for $p, q<0$ and $i<d$. So 2) and 4) are equivalent.

This last remark follows from 3). Note that by the Mayer-Vietoris sequence we have $\underline{H}_{\mathfrak{M}^{\Delta}}^{i}\left(T^{\Delta}\right)=\left(\underline{H}_{\mathfrak{M}^{+}}^{i}(T)\right)^{\Delta}=0$ for $i>d+1$, since $\operatorname{dim} T_{0, .}, \operatorname{dim} T T_{, 0}<d+2$.

2.6. Remark. Note the general fact that

$\inf \left\{i \mid \underline{H}_{\mathfrak{M}^{+}}^{i}(T) \neq 0\right\} \leq \mathrm{ht} \mathfrak{M}^{+} \leq d+2-\max \left(\operatorname{dim} T_{\cdot, 0} / \mathfrak{m} T_{\cdot, 0}, \operatorname{dim} T_{0, \cdot} / \mathfrak{m} T_{0, .}\right)$.

If $T$ is Cohen-Macaulay, it comes out from the proof of Theorem 2.5 that

$$
\left[\underline{H}_{\mathfrak{M}^{+}}^{i}(T)\right]_{p, q}= \begin{cases}{\left[\underline{H}_{\mathfrak{M}_{0, \cdot}}^{i}\left(T_{p, \cdot}\right)\right]_{q}} & \text { if } p \geq 0 \text { and } q<0, \\ {\left[\underline{H}_{\mathfrak{M}_{, 0}, 0}^{i}\left(T_{,, q}\right)\right]_{p}} & \text { if } p<0 \text { and } q \geq 0\end{cases}
$$

for $i<d+1$. Also recall that we then necessarily have $\left[\underline{H}_{\mathfrak{M}, 0}^{i}(T, q)\right]_{p}=0$ and $\left[\underline{H}_{\mathfrak{M}, 0}^{i}\left(T_{p, .}\right)\right]_{q}=0$ for all $p, q \geq 0$ and $i \geq 0$.

We now want to apply Theorem 2.5 to the case of multi-Rees algebras. Recall first the following fact ([6, Lemma 2.1]):

2.7. Lemma. Let $A$ be a local ring and let $I_{1}, \ldots, I_{r} \subset A$ be ideals of positive height. Then $a^{j}\left(R_{A}\left(I_{1}, \ldots, I_{r}\right)\right)=-1$ for all $j=1, \ldots, r$.

2.8. Corollary. Let $A$ be a local ring of dimension d and let $I, J \subset A$ be ideals of positive height. Let $\mathfrak{N}$ be the homogeneous maximal ideal of $R_{A}(I J)$. Then $R_{A}(I, J)$ is Cohen-Macaulay if and only if the following conditions hold:

a) $\left[\underline{H}_{\mathfrak{N}}^{i}\left(I^{p} R_{A}(I J)\right)\right]_{q}=0$ if $p \geq 0, q \notin\{-p, \ldots,-1\}$ and $i<d+1$;

b) $\left[\underline{H}_{\mathfrak{N}}^{i}\left(J^{q} R_{A}(I J)\right)\right]_{p}=0$ if $q \geq 0, p \notin\{-q, \ldots,-1\}$ and $i<d+1$.

Proof. The claim will follow from Theorem 2.53 ) if we can show that condition 3 ) c) holds in this case. Set $Q=R_{A}(I J)$. Let $p \geq 0$. There is an exact sequence

$$
0 \rightarrow K \rightarrow Q^{N} \rightarrow I^{p} Q \rightarrow 0
$$

of graded $Q$-modules. The corresponding long exact sequence of cohomology gives us an epimorphism

$$
\left(\underline{H}_{\mathfrak{N}}^{d+1}(Q)\right)^{N} \rightarrow \underline{H}_{\mathfrak{N}}^{d+1}\left(I^{p} Q\right) \rightarrow 0 .
$$

By Lemma 2.7 this implies $\left[\underline{H}_{\mathfrak{N}}^{d+1}\left(I^{p} Q\right)\right]_{q}=0$ for $q \geq 0$. Similarly, we also have $\left[\underline{H}_{\mathfrak{N}}^{d+1}\left(I^{q} Q\right)\right]_{p}=0$ for $p, q \geq 0$.

2.9. Example. Let $A$ be a local ring of dimension $d$ and let $I \subset A$ be an ideal of positive height. Put $T=R_{A}(I, I), R=R_{A}(I)$ and $Q=R_{A}\left(I^{2}\right)$. Let $\mathfrak{M}$ and $\mathfrak{N}$ be the homogeneous maximal ideals of $R$ and $Q$ respectively. Let $p \geq 0$. Noting that $I^{p} Q$ equals to the Veronesian $\left(I^{p} R\right)^{(2)}$ we get (cf. [9, Proposition (47.5)]) for all $i<d+1$ and $q \notin\{-p, \ldots,-1\}$ that $\left[\underline{H}_{\mathfrak{N}}^{i}\left(I^{p} Q\right)\right]_{q}=\left[\underline{H}_{\mathfrak{M}}^{i}\left(I^{p} R\right)\right]_{2 q}=\left[\underline{H}_{\mathfrak{M}}^{i}(R)\right]_{2 q+p}$ 
where the last step comes from the cohomology sequence corresponding to the exact sequence

$$
0 \rightarrow I^{p} R \rightarrow R(p) \rightarrow \bigoplus_{k=-p}^{-1} I^{k+p} t^{k} \rightarrow 0
$$

Similarly, $\left[\underline{H}_{\mathfrak{N}}^{i}\left(J^{q} Q\right)\right]_{p}=\left[\underline{H}_{\mathfrak{M}}^{i}(R)\right]_{2 p+q}$ for $p \notin\{-q, \ldots,-1\}$. By Corollary 2.8 we thus see that $T$ is Cohen-Macaulay if and only if $\left[\underline{H}_{\mathfrak{M}}^{i}(R)\right]_{p}=0$ for $p \neq-1$ and $i<d+1$. This can also be seen directly (cf. [6, Theorem 2.2]).

2.10. Corollary. Let $A$ be a local ring and let $I_{1}, \ldots, I_{r} \subset A$ be ideals of positive height. If $R_{A}\left(I_{1}, \ldots, I_{r}\right)$ is Cohen-Macaulay, also $R_{A}\left(I_{1} \cdots I_{r}\right)$ is Cohen-Macaulay.

Proof. Use induction on $r$. Put $B=R_{A}\left(I_{1}, \ldots, I_{r-2}\right)_{\mathfrak{N}}$ where $\mathfrak{N}$ is the homogeneous maximal ideal of $R_{A}\left(I_{1}, \ldots, I_{r-2}\right)$. We then have

$$
R_{A}\left(I_{1}, \ldots, I_{r}\right)_{\mathfrak{N}}=R_{B}\left(I_{r-1} B, I_{r} B\right) .
$$

We now obtain from Lemma 2.7 and Theorem 2.5 that

$$
R_{B}\left(\left(I_{r-1} B\right)\left(I_{r} B\right)\right)=R_{A}\left(I_{1}, \ldots, I_{r-2}, I_{r-1} I_{r}\right)_{\mathfrak{N}}
$$

is Cohen-Macaulay. Hence also $R_{A}\left(I_{1}, \ldots, I_{r-2}, I_{r-1} I_{r}\right)$ is Cohen-Macaulay. The induction hypothesis then implies that $R_{A}\left(I_{1} \cdots I_{r}\right)$ is Cohen-Macaulay.

The following example shows that the converse of Corollary 2.10 does not hold.

2.11. Example. The Cohen-Macaulayness of $R_{A}(I J)$ does not imply the CohenMacaulayness of $R_{A}(I, J)$ even if $R_{A}(I)$ and $R_{A}(J)$ are assumed to be CohenMacaulay. Let $(A, \mathfrak{m})$ be a local Cohen-Macaulay ring of positive dimension and let $I \subset A$ be an $\mathfrak{m}$-primary ideal with reduction number one. If $J \subset I$ is a minimal reduction, we thus have $I^{2}=I J$. Then $R_{A}(I), R_{A}(J)$ and $R_{A}(I J)=R_{A}\left(I^{2}\right)=$ $\left(R_{A}(I)\right)^{(2)}$ are Cohen-Macaulay (cf. [22, Proposition 3.1] and [21, Theorem 1.1]). Since also $\operatorname{gr}_{A}(I)$ is Cohen-Macaulay, the cohomology sequence corresponding to the exact sequence

$$
0 \rightarrow I R_{A}(I) \rightarrow R_{A}(I) \rightarrow g r_{A}(I) \rightarrow 0
$$

implies that $I R_{A}(I)$ and so also $I R_{A}\left(I^{2}\right)=\left(I R_{A}(I)\right)^{2}$ are Cohen-Macaulay modules. As $J R_{A}(I J)=J \oplus I^{3} \oplus I^{5} \oplus \cdots$, there is an exact sequence

$$
0 \rightarrow J R_{A}(I J) \rightarrow I R_{A}\left(I^{2}\right) \rightarrow I / J \rightarrow 0
$$

If $\mathfrak{N}$ denotes the homogeneous maximal ideal of $R_{A}(I J)$, the corresponding cohomology sequence then gives that

$$
\left[\underline{H}_{\mathfrak{N}}^{1}\left(J R_{A}(I J)\right)\right]_{0}=H_{\mathfrak{m}}^{0}(I / J)=I / J \neq 0 .
$$

According to Corollary 2.8 this means that $R_{A}(I, J)$ cannot be Cohen-Macaulay.

Although the converse of Corollary 2.10 does not hold, we will show in Theorem 2.13 that under certain assumptions the Cohen-Macaulayness of $R_{A}(I J)$ implies that of $R_{A}\left(I^{k}, J^{l}\right)$ for $k, l \gg 0$. First we need the following lemma:

2.12. Lemma. Let $T$ be a standard r-graded ring defined over local ring $(A, \mathfrak{m})$ which is a homomorphic image of a local Gorenstein ring B. Set $Z=\operatorname{Proj} T$ and $E=V_{+}(\mathfrak{m} T) \subset Z$. Then the following conditions are equivalent:

1) $Z$ is Cohen-Macaulay and equidimensional;

2) $H_{E}^{i}\left(Z, \mathcal{O}_{Z}(\mathbf{n})\right)=0$ for $i<\operatorname{dim} Z$ when $n_{1}, \ldots, n_{r} \ll 0$. 
Proof. Consider $Z$ as a closed subscheme of some multiprojective space $P=\mathbb{P}_{B}^{N_{1}} \times_{B}$ $\cdots \times{ }_{B} \mathbb{P}_{B}^{N_{r}}$. Let $\mathcal{I}$ be the ideal sheaf of $Z$ in $P$. According to the "local-global duality" of Lipman [13, Theorem p. 188]

$$
H_{E}^{i}\left(Z, \mathcal{O}_{Z}(\mathbf{n})\right)=\operatorname{Hom}_{B}\left(\operatorname{Ext}_{\mathcal{O}_{P}}^{\operatorname{dim} P-i}\left(\mathcal{O}_{P} / \mathcal{I}, \omega_{P}(-\mathbf{n})\right), E_{B}(k)\right)
$$

where $\omega_{P}$ is the dualizing sheaf of $P$. By using Theorem 1.6 we obtain from the spectral sequence of local to global Ext that if $n_{1}, \ldots, n_{r} \gg 0$, then

$$
\operatorname{Ext}_{\mathcal{O}_{P}}^{i}\left(\mathcal{O}_{P} / \mathcal{I}, \omega_{P}(\mathbf{n})\right)=\Gamma\left(P, \mathcal{E} x t_{\mathcal{O}_{P}}^{i}\left(\mathcal{O}_{P} / \mathcal{I}, \omega_{P}(\mathbf{n})\right)\right)
$$

for all $i \geq 0$. It follows that 2) is equivalent to having

$$
\Gamma\left(P, \mathcal{E} x t_{\mathcal{O}_{P}}^{i}\left(\mathcal{O}_{P} / \mathcal{I}, \omega_{P}\right)\right)(\mathbf{n})=0
$$

for $i>\operatorname{dim} P-\operatorname{dim} Z$ when $n_{1}, \ldots, n_{r} \gg 0$. This being in turn equivalent to

$$
\mathcal{E} x t_{\mathcal{O}_{P}}^{i}\left(\mathcal{O}_{P} / \mathcal{I}, \omega_{P}\right)=0
$$

for $i>\operatorname{dim} P-\operatorname{dim} Z$, we see that 2) is equivalent to $\operatorname{Ext}_{\mathcal{O}_{P, z}}^{i}\left(\mathcal{O}_{Z, z}, \mathcal{O}_{P, z}\right)=0$ for every closed point $z \in Z$ and $i>\operatorname{dim} \mathcal{O}_{P, z}-\operatorname{dim} Z$. By local duality this is the same as $\mathcal{O}_{Z, z}$ is Cohen-Macaulay and $\operatorname{dim} \mathcal{O}_{Z, z}=\operatorname{dim} Z$. If $Z^{\prime}$ is an irreducible component of $Z$ containing $z$, then also $\operatorname{dim} \mathcal{O}_{Z^{\prime}, z}=\operatorname{dim} Z$. Taking into account Lemma 1.2 we see that 1 ) and 2) are equivalent.

2.13. Theorem. Let $(A, \mathfrak{m})$ be a local ring and let $I, J \subset A$ be ideals of positive height. Set $X=\operatorname{Proj} R_{A}(I), Y=\operatorname{Proj} R_{A}(J)$ and $Z=\operatorname{Proj} R_{A}(I J)$. Let $f$ : $Z \rightarrow X$ and $g: Z \rightarrow Y$ be the canonical projections. Suppose that $R_{A}(I J)$ is Cohen-Macaulay. Then $R_{A}\left(I^{k}, J^{l}\right)$ is Cohen-Macaulay for all $k, l \gg 0$ if and only if the homomorphisms $\mathcal{O}_{X} \rightarrow f_{*} \mathcal{O}_{Z}, \mathcal{O}_{Y} \rightarrow g_{*} \mathcal{O}_{Z}$ are isomorphisms and we have $R^{i} f_{*} \mathcal{O}_{Z}=0, R^{i} g_{*} \mathcal{O}_{Z}=0$ for all $i>0$.

Proof. We may assume that $A$ is complete. Set $T=R_{A}(I, J), Z=\operatorname{Proj} T$ and $E=$ $V_{+}(\mathfrak{m} T) \subset Z$. Let $d=\operatorname{dim} A$. As $T^{\Delta}=R_{A}(I J)$ is equidimensional of dimension $d+1$, we know by Lemma 1.2 that $Z \cong Z^{\Delta}=\operatorname{Proj} R_{A}(I J)$ is equidimensional of dimension $d$. Since $T^{\Delta}$ is Cohen-Macaulay, we also see that $Z$ is Cohen-Macaulay. Lemma 2.12 therefore implies that $H_{E}^{i}\left(Z, \mathcal{O}_{Z}(p, q)\right)=0$ for $p, q \ll 0$ and $i<d$. By Theorem $1.6 H^{i}\left(Z, \mathcal{O}_{Z}(p, q)\right)=0$ for all $i>0$ when $p, q \gg 0$. As $T^{\Delta}$ is CohenMacaulay and $a\left(T^{\Delta}\right)<0$, we obtain from Lemma 2.3 that $\left[\underline{H}_{\left(T^{\Delta}\right)^{+}}^{i}\left(T^{\Delta}\right)\right]_{n}=0$ for all $n \geq 0$ and $i \geq 0$. This means, in particular, that $H^{i}\left(Z, \mathcal{O}_{Z}\right)=H^{i}\left(Z^{\Delta}, \mathcal{O}_{Z^{\Delta}}\right)=$ $\left[\underline{H}_{\left(T^{\Delta}\right)^{+}}^{i+1}\left(T^{\Delta}\right)\right]_{0}=0$ for all $i>0$. By the exact sequence

$$
0 \rightarrow\left[\underline{H}_{\left(T^{\Delta}\right)^{+}}^{0}\left(T^{\Delta}\right)\right]_{0} \rightarrow A \rightarrow \Gamma\left(Z^{\Delta}, \mathcal{O}_{Z^{\Delta}}\right) \rightarrow\left[\underline{H}_{\left(T^{\Delta}\right)^{+}}^{1}\left(T^{\Delta}\right)\right]_{0} \rightarrow 0
$$

we also see that the homomorphism $A \rightarrow \Gamma\left(Z, \mathcal{O}_{Z}\right)$ is an isomorphism. By Theorem 1.6 we know that the homomorphism $T_{p, q} \rightarrow \Gamma\left(Z, \mathcal{O}_{Z}(p, q)\right)$ is an isomorphism for $p, q \gg 0$. Let $k, l>0$. Set $Z^{(k, l)}=\operatorname{Proj} T^{(k, l)}$ where $T^{(k, l)}$ denotes the bi-Veronesian $\bigoplus_{p, q \geq 0} T_{p k, q l}$. There is an isomorphism $\varphi_{k, l}: Z \rightarrow Z^{(k, l)}$ such that $\varphi_{k, l}^{*}\left(\mathcal{O}_{Z^{(k, l)}}(p, q)\right)=\mathcal{O}_{Z}(p k, q l)$. It now follows from Theorem 2.54$)$ that $R_{A}\left(I^{k}, J^{l}\right)=T^{(k, l)}$ is Cohen-Macaulay for $k, l \gg 0$ if and only if the homomorphisms $T_{p, 0} \rightarrow \Gamma\left(Z, \mathcal{O}_{Z}(p, 0)\right), T_{0, q} \rightarrow \Gamma\left(Z, \mathcal{O}_{Z}(0, q)\right)$ are isomorphisms for $p, q \gg 0$ and $H^{i}\left(Z, \mathcal{O}_{Z}(p, 0)\right)=0, H^{i}\left(Z, \mathcal{O}_{Z}(0, q)\right)=0$ for $p, q \gg 0$ and $i>0$. For $p \gg 0$, we know that the homomorphism $T_{p, 0} \rightarrow \Gamma\left(X, \mathcal{O}_{X}(p)\right)$ is an isomorphism. As $f_{*}\left(\mathcal{O}_{Z}(p, 0)\right)=f_{*} \mathcal{O}_{Z} \otimes \mathcal{O}_{X}(p)$, we see that the homomorphism $T_{p, 0} \rightarrow \Gamma\left(Z, \mathcal{O}_{Z}(p, 0)\right)$ is an isomorphism for $p \gg 0$ if and only if the 
homomorphism $\mathcal{O}_{X} \rightarrow f_{*} \mathcal{O}_{Z}$ is an isomorphism. Similarly, the homomorphism $T_{0, q} \rightarrow \Gamma\left(Z, \mathcal{O}_{Z}(0, q)\right)$ is an isomorphism for $q \gg 0$ if and only if the homomorphism $\mathcal{O}_{X} \rightarrow g_{*} \mathcal{O}_{Z}$ is an isomorphism. By Lemma 2.1 the conditions $H^{i}\left(Z, \mathcal{O}_{Z}(p, 0)\right)=$ $0, H^{i}\left(Z, \mathcal{O}_{Z}(0, q)\right)=0$ for $p, q \gg 0$ and $i>0$ are equivalent to the conditions $R^{i} f_{*} \mathcal{O}_{Z}=0, R^{i} g_{*} \mathcal{O}_{Z}=0$ for $i>0$. This means that the claim has been proven.

Finally, we also want to mention the following application of Theorem 2.5.

2.14. Theorem. Let $S=A\left[X_{1}, \ldots, X_{r}, Y_{1}, \ldots, Y_{s}\right]$ be a polynomial ring over a local Cohen-Macaulay ring $A$. Let $I \subset S$ be a bihomogeneous ideal generated by forms of bidegree $(d, e)$ where $d, e>0$. Suppose that ht $i<\min (r / d, s / e)$. If the ring $S / I$ is Cohen-Macaulay, then also the diagonal subring $(S / I)^{\Delta}$ is Cohen-Macaulay.

Proof. Set $T=S / I$. We want to apply Theorem 2.5. As ht $I<\min (r, s)$, we have $\operatorname{dim} T \geq 2$ and $\operatorname{dim} T_{0,}, \operatorname{dim} T_{,, 0}<\operatorname{dim} T$. Therefore we need to show that $a^{1}(T)<0$ and $a^{2}(T)<0$. Consider, for example, the latter inequality. Let $\mathfrak{M}$ be the homogeneous maximal ideal of $S$. The $a$-invariant $a^{2}(T)$ does not change if we localize at the homogeneous maximal ideal $\mathfrak{M}_{\cdot, 0}$ of $S_{\cdot, 0}$ (cf. Lemma 1.1). We may therefore replace $S$ by the graded ring $B\left[Y_{1}, \ldots, Y_{s}\right]$ where $B$ is the localization of $S_{,, 0}$, and assume that $I \subset S$ is a homogeneous ideal generated by forms of degree $e$. Let $\mathfrak{n}$ be the maximal ideal of the local ring $B$. By moving to the faithfully flat extension $B[t]_{\mathfrak{n} B[t]}$ where $t$ is an indeterminate, we can assume that the residue field of $B$ is infinite. Put $h=$ ht $I$. Since $h=\operatorname{grade} I$, we can find forms $F_{1}, \ldots, F_{h} \in I_{e}$ such that $\left(F_{1}, \ldots, F_{h}\right)$ is a regular sequence (cf. [1, Proposition 1.5.12]). If $T^{\prime}=S /\left(F_{1}, \ldots, F_{h}\right)$, we have $\operatorname{dim} T^{\prime}=\operatorname{dim} T$ and there is an exact sequence $0 \rightarrow J \rightarrow T^{\prime} \rightarrow T \rightarrow 0$ of graded $S$-modules. The corresponding long exact sequence of cohomology then gives an epimorphism

$$
\underline{H}_{\mathfrak{M}}^{\operatorname{dim} T}\left(T^{\prime}\right) \rightarrow \underline{H}_{\mathfrak{M}}^{\operatorname{dim} T}(T) \rightarrow 0
$$

which implies that $a(T) \leq a\left(T^{\prime}\right)$. Since $T$ is Cohen-Macaulay and $\left(F_{1}, \ldots, F_{h}\right)$ is a regular sequence consisting of forms of degree $e$, we have $a\left(T^{\prime}\right)=a(S)+e h$ (cf. [23, Remark 5.1.21]). It is also well-known that $a(S)=-s$. Since $e h<s$ by assumption, we obtain $a(T) \leq a\left(T^{\prime}\right)<0$.

2.15. Example. Let $A$ be a local Cohen-Macaulay ring. Suppose that $F_{1}, \ldots, F_{s}$ are forms of degree $d$ in the polynomial ring $R=A\left[X_{1}, \ldots, X_{r}\right]$ such that $\left(F_{1}, \ldots, F_{s}\right)$ is a regular sequence. If $\mathfrak{A}$ denotes the ideal $\left(F_{1}, \ldots, F_{S}\right)$, it is then well-known that the Rees algebra $R_{R}(\mathfrak{A})$ is isomorphic to the quotient $S / I$ where $S$ is the polynomial ring $A\left[X_{1}, \ldots, X_{r}, Y_{1}, \ldots, Y_{s}\right]$ and $I$ is the ideal generated by the forms $F_{i} Y_{j}-F_{j} Y_{i}$ $(i, j=1, \ldots, s)$. Moreover, we know that $R_{R}(\mathfrak{A})$ is Cohen-Macaulay. Theorem 2.14 now says that if $(s-1) d<r$, then the diagonal subring $(S / I)^{\Delta}$ is also CohenMacaulay. In the case $A$ is a field we recover here a result of Simis, Trung and Valla (cf. [19]).

If $(s-1) d \geq r$, it may happen that $(S / I)^{\Delta}$ is not Cohen-Macaulay. Suppose, for example, that $A=k$ is a field, $r=2$ and $F_{1}=X_{1}^{3}, F_{2}=X_{2}^{3}$. Then

$$
S^{\Delta}=k\left[X_{1} Y_{1}, X_{1} Y_{2}, X_{2} Y_{1}, X_{2} Y_{2}\right]
$$


and

$$
\begin{aligned}
I^{\Delta}= & \left(X_{1} Y_{1}\right)^{2}\left(X_{1} Y_{2}\right)-\left(X_{2} Y_{1}\right)^{3}, \\
& \left(X_{2} Y_{1}\right)\left(X_{2} Y_{2}\right)^{2}-\left(X_{1} Y_{2}\right)^{3}, \\
& \left.\left(X_{1} Y_{1}\right)\left(X_{1} Y_{2}\right)^{2}-\left(X_{2} Y_{1}\right)^{2}\left(X_{2} Y_{2}\right)\right) .
\end{aligned}
$$

Using the isomorphism $k\left[Z_{0}, Z_{1}, Z_{2}, Z_{3}\right] /\left(Z_{1} Z_{2}-Z_{0} Z_{3}\right) \rightarrow S^{\Delta}$ where

$$
Z_{0} \mapsto X_{1} Y_{1}, Z_{1} \mapsto X_{1} Y_{2}, Z_{2} \mapsto X_{2} Y_{1}, Z_{3} \mapsto X_{2} Y_{2},
$$

we see that

$$
(S / I)^{\Delta}=k\left[Z_{0}, Z_{1}, Z_{2}, Z_{3}\right] /\left(Z_{1}^{3}-Z_{2} Z_{3}^{2}, Z_{2}^{3}-Z_{0}^{2} Z_{1}, Z_{0} Z_{1}^{2}-Z_{2}^{2} Z_{3}, Z_{0} Z_{3}-Z_{1} Z_{2}\right) .
$$

This is the homogeneous coordinate ring of the twisted quartic curve in $\mathbb{P}^{3}$, which is well-known to be a non-Cohen-Macaulay ring.

\section{An Application to the Joint REDUCtion NUmBers}

Let $T$ be a standard bigraded ring defined over a local ring. Let $z_{1}, \ldots, z_{\lambda} \in T$ be homogeneous elements. In analogy with the usual graded case (cf. [20]) we say that the sequence $\left(z_{1}, \ldots, z_{\lambda}\right)$ is filter-regular if

$$
\left[\left(z_{1}, \ldots, z_{i-1}\right): z_{i}\right]_{p, q}=\left[\left(z_{1}, \ldots, z_{i-1}\right)\right]_{p, q}
$$

for $p, q \gg 0(i=1, \ldots, \lambda)$. This is clearly equivalent to

$$
\left(z_{1}, \ldots, z_{i-1}\right): z_{i} \subset \bigcup_{n=0}^{\infty}\left(z_{1}, \ldots, z_{i-1}\right):\left(T^{+}\right)^{n} \quad(i=1, \ldots, \lambda) .
$$

Recall that a graded ring defined over a local ring is said to be generalized CohenMacaulay if its localization at the homogeneous maximal ideal is a generalized Cohen-Macaulay ring. For information about generalized Cohen-Macaulay rings we refer to [18] and [9].

3.1. Lemma. Let $T$ be a standard bigraded ring defined over an Artinian local ring. Suppose that $T^{\Delta}$ is a generalized Cohen-Macaulay ring. Let $\left(z_{1}, \ldots, z_{\lambda}\right)$ be a system of parameters of $T^{\Delta}$ consisting of homogeneous elements of degree one. Then $\left(z_{1}, \ldots, z_{\lambda}\right)$ is a filter-regular sequence on $T$. Suppose, moreover, that we have $z_{i}=x_{i} y_{i}(i=1, \ldots, \lambda)$ for some homogeneous elements $x_{1}, \ldots, x_{\lambda}, y_{1}, \ldots, y_{\lambda} \in T$. Then also $\left(x_{1}, \ldots, x_{\lambda}\right)$ and $\left(y_{1}, \ldots, y_{\lambda}\right)$ are filter-regular sequences on $T$.

Proof. Let $i \in\{1, \ldots, \lambda\}$. It is enough to show that

$$
\left(\left(z_{1}, \ldots, z_{i-1}\right): z_{i}\right)_{P}=\left(z_{1}, \ldots, z_{i-1}\right)_{P}
$$

for $P \in \operatorname{Proj} T$. If $\left(z_{1}, \ldots, z_{i}\right)_{P}=(1)$, we are done. Assume thus $\left(z_{1}, \ldots, z_{i}\right)_{P} \neq(1)$. The localization $\left(T^{\Delta}\right)_{P \Delta}$ is now Cohen-Macaulay. Since $T^{\Delta}$ is equidimensional and catenary, $\left(z_{1} / 1, \ldots, z_{i} / 1\right)$ must be a part of a system of parameters of $\left(T^{\Delta}\right)_{P}$. Therefore it is also a regular sequence. Recall that for any standard $r$-graded ring $T$ and a homogeneous prime ideal $P \subset T, T_{P} \cong\left(T_{(P)}\left[t_{1}, \ldots, t_{r}\right]\right)_{\mathfrak{P}}$, where $t_{1}, \ldots, t_{r}$ are indeterminates and $\mathfrak{P}=P_{(P)} T_{(P)}\left[t_{1}, \ldots, t_{r}\right]$ (cf. [9, Corollary (12.18)] for the case $r=1)$. Choose $s \in T_{1,1}, s \notin P$. Then also $\left(z_{1} / s, \ldots, z_{i} / s\right)$ is a regular sequence in $\left(T^{\Delta}\right)_{P^{\Delta}}$. Since $\left(T^{\Delta}\right)_{\left(P^{\Delta}\right)} \rightarrow\left(T^{\Delta}\right)_{P^{\Delta}}$ is a faithfully flat extension, it is a regular sequence also in $\left(T^{\Delta}\right)_{\left(P^{\Delta}\right)}=T_{(P)}$. Because $T_{(P)} \rightarrow T_{P}$ is a faithfully flat extension, it follows that $\left(z_{1} / s, \ldots, z_{i} / s\right)$ and so also $\left(z_{1} / 1, \ldots, z_{i} / 1\right)$ are regular sequences in $T_{P}$. This proves the first assertion. The second one follows from the fact that 
if $\left(x_{1} y_{1} / 1, \ldots, x_{i} y_{i} / 1\right)$ is a regular sequence in $T_{P}$, then so are $\left(x_{1} / 1, \ldots, x_{i} / 1\right)$ and $\left(y_{1} / 1, \ldots, y_{i} / 1\right)$ (unless $\left(x_{1} / 1, \ldots, x_{i} / 1\right)=(1)$ or $\left.\left(y_{1} / 1, \ldots, y_{i} / 1\right)=(1)\right)([10$, Exercise 12, p. 102]).

We next recall certain definitions from [11]. Let us say that an ideal $\mathfrak{A} \subset T$ is irrelevant if $\mathfrak{A}_{p, q}=T_{p, q}$ for $p, q \gg 0$. Suppose $x_{1}, \ldots, x_{\lambda} \in T_{1,0}$ and $y_{1}, \ldots, y_{\lambda} \in$ $T_{0,1}$. The set $\left\{x_{1}, \ldots, x_{\lambda}, y_{1}, \ldots, y_{\lambda}\right\}$ is called a complete reduction of $T$ if the ideal $\left(x_{1} y_{1}, \ldots, y_{\lambda} y_{\lambda}\right) \subset T$ is irrelevant. A joint reduction of type $(\mu, \nu)(\mu+\nu=$ $\lambda)$ is a set $\left\{u_{1}, \ldots, u_{\mu}, v_{1}, \ldots, v_{\nu}\right\}$ with $u_{1}, \ldots, u_{\mu} \in T_{1,0}$ and $v_{1}, \ldots, v_{\nu} \in T_{0,1}$ such that the ideal $\left(u_{1}, \ldots, u_{\mu}, v_{1}, \ldots, v_{\nu}\right) \subset T$ is irrelevant. From a complete reduction $\left\{x_{1}, \ldots, x_{\lambda}, y_{1}, \ldots, y_{\lambda}\right\}$ we can always form joint reductions of any type: if $\left\{i_{1}, \ldots, i_{\mu}\right\} \cup\left\{j_{1}, \ldots, j_{\nu}\right\}$ is a partition of the set $\{1, \ldots, \lambda\}$ into two disjoint subsets, then $\left\{x_{i_{1}}, \ldots, x_{i_{\mu}}, y_{j_{1}}, \ldots, y_{j_{\nu}}\right\}$ is a joint reduction of $T$ of type $(\mu, \nu)$. By definition the analytic spread of $T^{\Delta}$ is $l\left(T^{\Delta}\right)=\operatorname{dim} T^{\Delta} / \mathfrak{n} T^{\Delta}$ where $\mathfrak{n}$ denotes the maximal ideal of $B=T_{\mathbf{0}}$. According to [11, Theorem 1.6, Lemma 1.7] there always exists complete reductions of $T$ with $\lambda \geq l\left(T^{\Delta}\right)$ if the residue field of $B$ is infinite. It is well-known that this can be achieved by moving to the faithfully flat extension $B[t]_{\mathfrak{n} B[t]}$ where $t$ is an indeterminate.

3.2. Definition. Let $T$ be a standard bigraded ring defined over a local ring. Let $K=\left\{x_{1}, \ldots, x_{\lambda}, y_{1}, \ldots, y_{\lambda}\right\}$ be a complete reduction of $T$. Suppose $\mu, \nu \in$ $\{1, \ldots, \lambda\}$ with $\lambda+\nu=\lambda$. Let $n$ be the smallest integer such that

$$
\left[\left(x_{i_{1}}, \ldots, x_{i_{\mu}}, y_{j_{1}}, \ldots, y_{j_{\nu}}\right)\right]_{p, q}=T_{p, q}
$$

for $p, q>n$ and all partitions $\left\{i_{1}, \ldots, i_{\mu}\right\} \cup\left\{j_{1}, \ldots, j_{\nu}\right\}$ of $\{1, \ldots, \lambda\}$. We call $n$ the joint reduction number of type $(\mu, \nu)$ with respect to $K$ and denote it by $r_{K}^{\mu, \nu}(T)$.

Let $A$ be a local ring and let $I, J \subset A$ be ideals. Let $G$ be the bi-form $\operatorname{ring} g r_{A}(I, J)=R_{A}(I, J) /(I J) R_{A}(I, J)$. If $a_{1}, \ldots, a_{\lambda} \in I, b_{1}, \ldots, b_{\lambda} \in J$, let $a_{1}^{*}, \ldots, a_{\lambda}^{*} \in G_{1,0}, b_{1}^{*}, \ldots, b_{\lambda}^{*} \in G_{0,1}$ denote the corresponding initial forms. We then say that $K=\left\{a_{1}, \ldots, a_{\lambda}, b_{1}, \ldots, b_{\lambda}\right\}$ is a complete reduction of $I$ and $J$ if $K^{*}=\left\{a_{1}^{*}, \ldots, a_{\lambda}^{*}, b_{1}^{*}, \ldots, b_{\lambda}^{*}\right\}$ is a complete reduction of $G$. In a similar way we speak about joint reductions of $I$ and $J$. By definition the joint reduction number $r_{K}^{\mu, \nu}(I, J)=r_{K^{*}}^{\mu, \nu}(G)$ is now the smallest integer $n$ satisfying

$$
I^{n+1} J^{n+1}=\left(a_{i_{1}}, \ldots, a_{i_{\mu}}\right) I^{n} J^{n+1}+\left(b_{j_{1}}, \ldots, b_{j_{\nu}}\right) I^{n+1} J^{n}
$$

for all partitions $\left\{i_{1}, \ldots, i_{\mu}\right\} \cup\left\{j_{1}, \ldots, j_{\nu}\right\}$ of $\{1, \ldots, \lambda\}$.

3.3. Theorem. Let $T$ be a standard bigraded ring defined over an Artinian local ring. Suppose that $T^{\Delta}$ is a generalized Cohen-Macaulay ring of dimension $d$ and that $\left[\underline{H}_{T^{+}}^{i}(T)\right]_{p, q} \geq 0$ for all $p, q \geq 0$ and $i \geq 0$. Let $K=\left\{x_{1}, \ldots, x_{d}, y_{1}, \ldots, y_{d}\right\}$ be a complete reduction of $T$. We then have $r_{K}^{\mu, \nu}(T) \leq \max (\mu, \nu)-1$ for all $\mu, \nu \in$ $\{1, \ldots, d\}$ with $\mu+\nu=d$.

Proof. Consider first any filter-regular sequence $\left(z_{1}, \ldots, z_{\lambda}\right)$ on $T$ consisting of homogeneous elements. Suppose that $z_{j}$ is of degree $\left(p_{j}, q_{j}\right)$. Set $T^{j}=T /\left(z_{1}, \ldots, z_{j-1}\right)$ $(j=1, \ldots, \lambda+1)$. We have for $j=1, \ldots, \lambda$ the exact sequences

$$
0 \rightarrow K^{j} \rightarrow T^{j} \rightarrow T^{j} / K^{j} \rightarrow 0
$$

and

$$
0 \rightarrow T^{j} / K^{j}\left(-p_{j},-q_{j}\right) \stackrel{\cdot z_{j}}{\longrightarrow} T^{j} \rightarrow T^{j+1} \rightarrow 0
$$


where $K^{j}=\left(z_{1}, \ldots, z_{j-1}\right): z_{j} /\left(z_{1}, \ldots, z_{j-1}\right)$. Because

$$
K^{j} \subset\left(z_{1}, \ldots, z_{j-1}\right):\left(T^{+}\right)^{n} /\left(z_{1}, \ldots, z_{j-1}\right)
$$

for $n \gg 0$, the long exact sequence of cohomology corresponding to the first sequence implies that $\underline{H}_{T^{+}}^{i}\left(T^{j} / K^{j}\right)=\underline{H}_{T^{+}}^{i}\left(T^{j}\right)$ for $i>0$. The long exact sequence of cohomology corresponding to the second sequence gives for all $i \geq 0$ the exact sequence

$$
\left[\underline{H}_{T^{+}}^{i}\left(T^{j}\right)\right]_{p, q} \rightarrow\left[\underline{H}_{T^{+}}^{i}\left(T^{j+1}\right)\right]_{p, q} \rightarrow\left[\underline{H}_{T^{+}}^{i+1}\left(T^{j}\right)\right]_{p-p_{j}, q-q_{j}} .
$$

By assumption $\left[\underline{H}_{T^{+}}^{i}(T)\right]_{p, q}=0$ for $p, q \geq 0$. It follows by induction on $j$ that $\left[\underline{H}_{T^{+}}^{i}\left(T^{j}\right)\right]_{p, q}=0$ for $p \geq p_{1}+\cdots+p_{j-1}$ and $q \geq q_{1}+\cdots+q_{j-1}$. In particular, we obtain $\left[\underline{H}_{T^{+}}^{0}\left(T^{\lambda+1}\right)\right]_{p, q}=0$ for $p \geq p_{1}+\cdots+p_{\lambda}$ and $q \geq q_{1}+\cdots+q_{\lambda}$.

Let $\mu, \nu \in\{1, \ldots, d\}$ with $\mu+\nu=d$ and let $\left\{i_{1}, \ldots, i_{\mu}\right\} \cup\left\{j_{1}, \ldots, j_{\nu}\right\}$ be a partition of $\{1, \ldots, d\}$. As $x_{1} y_{1}, \ldots, x_{d} y_{d}$ is a system of parameters of $T^{\Delta}$, we obtain from Lemma 3.1 that $\left(x_{i_{1}}, \ldots, x_{i_{\mu}}, y_{j_{1}}, \ldots, y_{j_{\nu}}\right)$ is a filter-regular sequence on $T$. We now apply the result proved above to this sequence. So $\left[\underline{H}_{T^{+}}^{0}\left(T^{d+1}\right)\right]_{p, q}=0$ for $p \geq \mu$ and $q \geq \nu$. On the other hand, if $r=r_{K}^{\mu, \nu}(T)$, we must have $\left[\underline{H}_{T^{+}}^{0}\left(T^{d+1}\right)\right]_{r, r}=$ $T_{r, r}^{d+1} \neq 0$. Thus $r<\mu$ or $r<\nu$.

3.4. Corollary. Let $(A, \mathfrak{m})$ be a local ring of dimension $d$ and let $I, J \subset A$ be $\mathfrak{m}$-primary ideals. Let $K=\left\{a_{1}, \ldots, a_{d}, b_{1}, \ldots, b_{d}\right\}$ be a complete reduction of $I$ and $J$. If $R_{A}(I, J)$ is Cohen-Macaulay, then $r_{K}^{\mu, \nu}(I, J) \leq \max (\mu, \nu)-1$ for all $\mu, \nu \in\{1, \ldots, d\}$ with $\mu+\nu=d$.

Proof. Set $T=R_{A}(I, J)$ and $G=g r_{A}(I, J)=T /(I J) T$. By Corollary $2.10 R_{A}(I J)$ is Cohen-Macaulay. Therefore $G^{\Delta}=g r_{A}(I J)$ is generalized Cohen-Macaulay (cf. $\left[21\right.$, Proposition 3.3]). Let us show that $\left[\underline{H}_{G^{+}}^{i}(G)\right]_{p, q}=0$ for all $p, q \geq 0$ and $i \geq 0$. Consider the exact sequences

$$
0 \rightarrow T^{+} \rightarrow T \rightarrow T / T^{+} \rightarrow 0
$$

and

$$
0 \rightarrow T^{+}(1,1)=(I J) T \rightarrow T \rightarrow G \rightarrow 0 .
$$

As $\underline{H}_{T^{+}}^{0}\left(T / T^{+}\right)=T / T^{+}$and $\underline{H}_{T^{+}}^{i}\left(T / T^{+}\right)=0$ for $i>0$, the cohomology sequence corresponding to the first sequence gives $\left[\underline{H}_{T^{+}}^{i}\left(T^{+}\right)\right]_{p, q}=\left[\underline{H}_{T^{+}}^{i}(T)\right]_{p, q}$ for all $i \geq 0$ if $p \neq 0$ and $q \neq 0$. We then obtain from the cohomology sequence of the second sequence for all $p, q \geq 0$ and $i \geq 0$ the exact sequence

$$
\left[\underline{H}_{T^{+}}^{i}(T)\right]_{p, q} \rightarrow\left[\underline{H}_{G^{+}}^{i}(G)\right]_{p, q} \rightarrow\left[\underline{H}_{T^{+}}^{i+1}(T)\right]_{p+1, q+1} .
$$

Since by Lemma 2.4 and Theorem 2.52$)\left[\underline{H}_{T^{+}}^{i}(T)\right]_{p, q}=0$ for all $p, q \geq 0$ and $i \geq 0$, we get that also $\left[\underline{H}_{G^{+}}^{i}(G)\right]_{p, q}=0$ for all $p, q \geq 0$ and $i \geq 0$. We can now apply Theorem 3.3 to get the claim.

Let $(A, \mathfrak{m})$ be a two-dimensional local Cohen-Macaulay ring and let $I, J \subset A$ be $\mathfrak{m}$-primary ideals. Verma proved in [24, Theorem 3.2] that $r_{K}^{1,1}(I, J)=0$ for all complete reductions $K=\left\{a_{1}, a_{2}, b_{1}, b_{2}\right\}$ of $I$ and $J$ if and only if the formula $e_{1}(I, J)=l(A / I J)-l(A / I)-l(A / J)$ holds for the mixed multiplicity $e_{1}(I, J)$ of $I$ and $J$. In this case [8, Theorem 3.4] says that the Cohen-Macaulayness of $R_{A}(I, J)$ is equivalent to that of $R_{A}(I)$ and $R_{A}(J)$. Combining these results with Corollary 3.4 gives us 
3.5. Corollary. Let $(A, \mathfrak{m})$ be a local Cohen-Macaulay ring of dimension two and let $I, J \subset A$ be $\mathfrak{m}$-primary ideals. Then $R_{A}(I, J)$ is Cohen-Macaulay if and only if $R_{A}(I), R_{A}(J)$ are Cohen-Macaulay and we have that $e_{1}(I, J)=l(A / I J)-l(A / I)-$ $l(A / J)$.

\section{REFERENCES}

1. W. Bruns and J. Herzog, Cohen-Macaulay rings, Cambridge University Press, Cambridge, 1993. MR 95h:13020

2. S. Goto and K. Watanabe, On graded rings I, J. Math. Soc. Japan 30 (1978), 179-213. MR 81m:13021

3. S. Goto and K. Watanabe, On graded rings II, Tokyo J. Math. 1 (1978), 237-260. MR 81m:13022

4. A. Grothendieck and J. Dieudonné, Eléments de Géométrie Algébrique II, Publ. Math. I.H.E.S. 8 (1961). MR 36:177b

5. R. Hartshorne, Residues and Duality, Springer Lecture Notes 20, Springer-Verlag, Berlin, Heidelberg, New York, 1966. MR 36:5145

6. M. Herrmann, E. Hyry, and J. Ribbe, On the Cohen-Macaulay and Gorenstein properties of multi-Rees algebras, Manuscripta Math. 79 (1993), 343-377. MR 94h:13003

7. M. Herrmann, E. Hyry, and J. Ribbe, On multi-Rees algebras (with an appendix by $N$. $V$. Trung), Math. Ann. 301 (1995), 249-279. MR 96c:13005

8. M. Herrmann, E. Hyry, J. Ribbe, and Z. Tang, Reduction numbers and multiplicities of multigraded structures, J. Algebra 197 (1997), 311-341. MR 98k:13006

9. M. Herrmann, S. Ikeda, and U. Orbanz, Equimultiplicity and blowing up, Springer-Verlag, Berlin, Heidelberg, New York, 1988. MR 89g:13012

10. I. Kaplansky, Commutative rings, Allyn and Bacon, Boston, 1970. MR 40:7234

11. D. Kirby and D. Rees, Multiplicities in graded rings I: The general theory, Contemp. Math. 159 (1994), 209-267. MR 95b:13002

12. S. Kleiman and A. Thorup, A geometric theory of the Buchsbaum-Rim multiplicity, J. Algebra 167 (1994), 168-231. MR 96a:14007

13. J. Lipman, Desingularization of two-dimensional schemes, Ann. of Math. 107 (1978), 151207. MR 58:10924

14. J. Lipman, Cohen-Macaulayness in graded algebras, Math. Res. Letters 1 (1994), 149-157. MR 95d:13006

15. D. G. Northcott and D. Rees, Reduction of ideals in local rings, Proc. Cambridge Philos. Soc. 50 (1954), 145-158. MR 15:596a

16. L. O'Carroll, On two theorems concerning reductions in local rings, J. Math. Kyoto Univ. 27 (1987), 61-67. MR 87m:13034

17. D. Rees, Generalizations of reductions and mixed multiplicities, J. London Math. Soc. (2) 29 (1984), 397-414. MR 86e:13023

18. P. Schenzel, N. V. Trung, and N. T. Cuong, Verallgemeinerte Cohen-Macaulay Moduln, Math. Nachr. 85 (1978), 57-73. MR 80i:13008

19. A. Simis, The diagonal subalgebra of a multigraded $k$-algebra, in Commutative Algebra: extended abstracts of an international conference, July 27-August 1, 1994, Vechta, Germany (ed. by Winfried Bruns), Vechtaer Universitätsschriften 13, Runge, Cloppenburg, 1994.

20. N. V. Trung, Reduction exponent and degree bound for the defining equations of graded rings, Proc. Amer. Math. Soc. 101 (1987), 229-237. MR 89i:13031

21. N. V. Trung and S. Ikeda, When is the Rees algebra Cohen-Macaulay?, Comm. Algebra 17 (1989), 2893-2922. MR 91a:13009

22. P. Valabrega and G. Valla, Form rings and regular sequences, Nagoya Math. J. 72 (1978), 93-101. MR 80d:14010

23. W. V. Vasconcelos, Arithmetic of Blowup Algebras, LMS Lecture Note Ser. 195, Cambridge University Press, Cambridge, 1994. MR 95g:13005

24. J. K. Verma, Joint reductions of complete ideals, Nagoya Math. J. 118 (1990), 155-163. MR 91c: 13018 
25. J. K. Verma, Joint reductions and Rees algebras, Math. Proc. Cambridge Philos. Soc. 109 (1991), 335-343. MR 92h:13007

26. J. K. Verma, Multigraded Rees algebras and mixed multiplicities, J. Pure Appl. Algebra 77 (1992), 219-228. MR 93e: 13005

National Defence College, Santahamina, Fin-00860 Helsinki, Finland

E-mail address: eero.hyry@helsinki.fi 